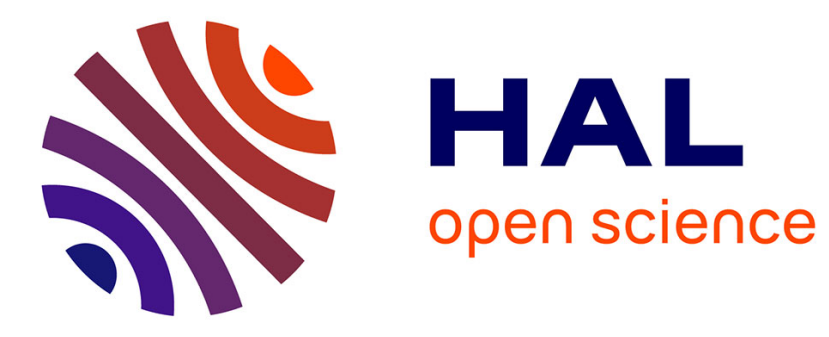

\title{
Philosophie de la neuropsychologie du nombre
}

\author{
Hourya Benis Sinaceur
}

\section{To cite this version:}

Hourya Benis Sinaceur. Philosophie de la neuropsychologie du nombre. Intellectica - La revue de l'Association pour la Recherche sur les sciences de la Cognition (ARCo), 2014, 62, pp.103-144. halshs01122609

\section{HAL Id: halshs-01122609 \\ https://shs.hal.science/halshs-01122609}

Submitted on 4 Mar 2015

HAL is a multi-disciplinary open access archive for the deposit and dissemination of scientific research documents, whether they are published or not. The documents may come from teaching and research institutions in France or abroad, or from public or private research centers.
L'archive ouverte pluridisciplinaire HAL, est destinée au dépôt et à la diffusion de documents scientifiques de niveau recherche, publiés ou non, émanant des établissements d'enseignement et de recherche français ou étrangers, des laboratoires publics ou privés. 
Philosophie de la neuropsychologie du nombre

\section{Hourya BENIS SINACEUR*}

Résuméc. Comment le cerveau et l'esprit appréhendent-ils la dimension quantitative et numérique des événements el des phénomènes du monde ?

Les neurosciences cognitives produisent une myriade de résultats expérimentaux qui éclairent d'un nouveau jour les processus numériques, s.spposés primitifs, du cerveau et de l'esprit.

Cependant, les hypothèses et les modalités de réalisation des expériencess, done les résultats, sont conditionnés par les modèles mathématiques et informatiques servant a rassembler et expliquer les données et ne sont pas immunisées contre les parti-pris phïlosophiques culturellement constitués et spontanénent ou délibérénent assumés.

Le but de cet article est de mettre en évidence les infrastructures technigues et idéologiques de la recherche de fondements effectifs, neuraux on cognitits, matériels ou symboliques, pour nos capacités arithmétiques élémentaires

Mots-clés: nombre. cognition arithmétique, perception, catégorisation, continu, discret, image mentale, index, symbole, schéna, schème, intuition, concept, objet.

ABstraCt. Neuropsychology of numbers: philosophical remarks. How do we extract numbers from our perceiving the surrounding world?

Neurosciences and cognitive sciences provide us with a myriad of empirical findings that shed light on hypothesized primitive numerical processes in the brain and in the mind.

Yet, the hypotheses based on which the experiments are conducred, hence the results, depend strongly on sophisticated mathematical models. These sophisticated models are used to describe and explain neural data or cognitive representations that supposedly are the roots of primary arithmetical activity. Moreover philosophical previews are involved in the generalizations presented as extrapolations from experimental data.

My aim is at bringing to light the lechnical and conceptual infrastructures of the presumed netral or cognitive, material or symbolic, actual fouthdations for our elementary arithmetical abilities.

Keywords: number, arithmetical cognition, perception, categorization, continum, discrete, mental image, index, symbol, schema, scheme, intuition. concept, object.

Depuis plusieurs décennies, nos aptitudes numériques font l'objet d'une multitude impressionnante de recherches expérimentales de divers types. Cet engagement expérimental correspond ali fait que la question posée n"est pas "qu'est-ce qu'un nombre? "» mais "comment le cerveal et l'esprit appréhendent-ils la dimension quantitative et numérique des événements et des

- Directrice de recherche émérite Institut d'Histoire et Ptilosophie des Sciences at des Techniques (IHPST), Universitế Paris I Panthéon-Sorbonne-CNRS-ENS Ulm, sinzceureatscanoe ens. fr 
phénomènes du monde ? », Les réponses au comment n'excluent cependant pas de renouveler les conceptions sur \&la nature » ou «l'origine » des nombres.

On peut distinguer deux types d'expérimentation selon qu'elles son focalisées sur l'observation du fonctionnement du cerveau ou sur l'observation du fonctionnement de l'esprit : d'un côté exploration neurobiologique ou neurophysiologique avec, en particulier, interprétation d'images du cerveau fournies par les apparéls à résonnance magnêtique nucléaire, de l'autre ictentification de processus mentaux par l'étude expérimentale du comportement observable de sujets aninaux ou humains exposés à des protocoles impliquant des quantités ou des nombres. Le premier type de méthode établit des cartes géographiques du cerveat représentant les zones activées lors de l'exécution de certaines tâches, le deuxième rejette la réduction behaviouriste de l'activité psychique au comportement et imagine des protocoles fondés sur l'habituation et la réaction à la nouveauté ou à la violation de lois physiques pour identifier, par le biais de dispositifs vistels, auditifs ou tactiles, les images ou operations mentales sous-tendant les comportements répondant aux tâches proposées. Le plus souvent des méthodes des deux types sont combinées entre elles et avec d'autres', comme l'étude neuropsychologique des déficits de compétence en liaison avec des lésions đu cerveau et létablissement de modèles computationnels des fonctions cognitives, en vue d'obtenir une explication simultanément neurophysique et psycho-physiologique des processus mentaux engagés dans nos réactions à des stimuli incitant à discerner, comparer. ajouter ou soustraire des quantités. Beaucoup d"hypothèses validées pal une méthode le sont par d'autres. et celles qui ne le sont pas foumissent tant aux neurobiologistes qu' aux spécialistes de psychologie cognitive expérimentale matière à des recherches interdisciplinaires articulées sur des hypothèses plus ramifiées et de mieux en mieux contextualisées. L affinement des résultats est toujours en marche et semble ne pas devoir $s$ arrêtel, tandis que leur mise en perspective socio-historique se fait de plus en plus insistante.

Je me propose dans cet article de regarder avec les yeux d'une philosoplae une masse non négligeable de résultats consignés dans les publications d'experts en neurosciences et en sciences cognitives, en m'attardant sur leurs hypothèses. explicites ou non. et sur les conclusions parfois restreintes ou prudentes mais souvent irrésistiblement proposées comme éléments générateurs de conceptions théoriques générales de la cognition. Je me limite aux travaux et publications pontant sur notre manière d'appréhender ou de

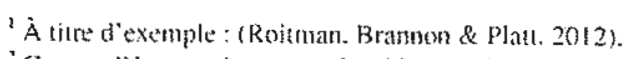

: Ces motêles corsistert en algonithunts génériqués permeltant de comprendre les relations complexes entre la strucurec el les fonctions du cerveau. Ils s'appuient sur une théotie fonctionnaliste qui compare

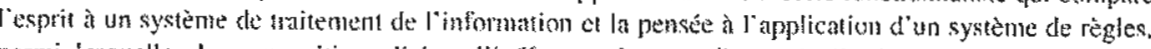

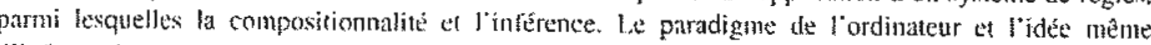

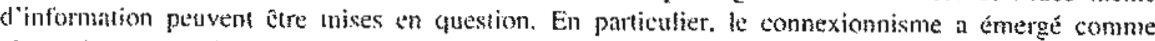

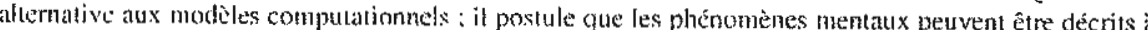

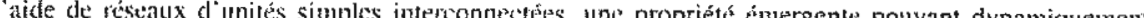
apparaitre à certains degrés de conplexités. construire la dimension quantitative et numérique des événements et phénomènes du monde.

Je montre que les recherches expérimentales sur la cognition mathématique, en utilisant - avec un certain bon droit - des modèles de recueil et d'explication des données foumis par les mathématiques modernes ou contemporaines, incorporent inévitablement un parti-pris sur la nature de l'objet mathématique et sur le fondement de nos capacités arithmétiques.

Je montre que le recours pré- ou post-expérimental à des fragments de doctrines philosophiques en vue de donner une dimension théorique aux constructions de faits d'expérience passe souvent par une distorsion involontaire de ces doctrines sous prétexte de leur adaptation délibérée aux données des sciences d'aujourd'hui.

Je constate que bien des expérimentateurs tiennent à la perspective fondationnelle, poursuivant la chimère de la base ou origine première, lors

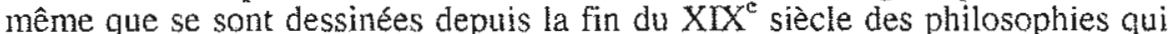
démoncent l'illusion du fondement et du commencement originel (Wittgenstein, Cavaillès), des philosophies dialectiques et immanentistes plutôt que réductionnistes qui intègrent les conditions historiques et contextuelles de la connaissance comme paramètre dans l'analyse structurale des connaissances (héritage croisé de Spinoza, Hegel, Marx et Nietzsche où prévaut le devenir sur l'être et le processus sur l'état, chez des auteuis aussi différents que Whitehead, Prigogine, Deuleuze ou Foucault) et que se multiplient aujourd'hui les travaux scientifiques (physiques, biologiques, psychologiques, etc.) attentifs aux processus et à leurs dynamiques plutôt qu'à leur origine unilinéaire ou a leurs causes indépendantes?

\section{I - LE NATIVISME}

Je commence par rappeler brièvement le cadre général plus ou moins généralement accepté par nombre d'experts contemporains en sciences cognitives. Il s'agit d'un rappel, non d'une valorisation personnelle de ce cadre, et bien entendu les dérives réductionnistes qui en résultent au nom d'un matêrialisme déterministe sans faille sont fortement sujettes à caution ${ }^{4}$.

L'expérimentation a révélé que les très jeunes enfants et certains animaux ont une aptitude numérique spontanée, c'est-à-dire non apprise, qui se manifeste de manière à la fois beaucoup plus précoce et plus flexible que les travaux de Piaget ne l'avaient laissé supposer. Neurobiologistes et psychologutes s'accordent généralement à penser que les aptitudes numériques

"Je renvoie à l"ensernble des artictes da numéro 16 de Intellecrica, intitulé Biologie el Cogninion. 1993. et notamment it ceivi de B.C. Goodwin. qui défend l'idé que l'espcit et le cerveau sont deux faces de processus dynaraiques en relation circulaire et à celui de J. Stewart qui dénonce le caracic̀ce jdéologique du nativisıe en lui préférant une perspective construetiviste. qui me paraît. en effat. plus pertinente. Voir l'analyse te (Stewart, 1993)

Jean Piaget présente le développement de l'enfant conutne uné accurnułation linénire conmençant aus stade sensorimotcur (de 0 a 2 ans) et parcourant un stade préopisatoire (de 2 à 6 anks), puis opératoire avec des opérations concrètes (de 6 à 10 ans) pour artiver au stade des opérations abstraites (de 10 à 16 ans). Le nombre se consiruit par une synthèse logico-mathématique d'opérations telles que la classification, la sériation. ha conservation mémoniele de quantités discrètes. 
sont «précâblées » à la naissance et ils promeuvent ainsi, de mantère plus ou moins forte ou nuancée, une perspective innéiste ou nativiste, opposée à lempirisme "classique " d"un John Locke pour qui notre cerveatl est, à la naissance. une tabula rasa et développe ses facultés par interactions avec l'environnement en établissant progressivement des associations et corrélations entre phénomènes perçus.

Le nativisme est cerainement un nouvel empirisme dans la mesure oè i] soutient une conception physicaliste ou mentaliste des processus de connaissance tout en tenant compte du développement des équipements innés par expériences et apprentissage. Il s"agit de monter des protocoles d'expétiences relativement contrốlables et reproductibles qui permettent de vérifier ou d'inférer une corrélation entre un phénomène cérébrat ou un comportement et un acte de comprétaension par un sujet animal ou hamain, enfant ou adulte. Mais le nativisme est aussi un nouveau rationalisme en tant qu'il défend l'hypothèse de structures cognitives universelles incarnées dans larchitecture cérébrale, plus l'hypothèse de mécanismes perceptuels et conceptuels innés. er par conséquent l'existence dès les premiers jours de vie de représenations structurées ayant un contenu relativement abstrait, comme c'est le cas pour les contenus numériques. On peut certainement parler ici de rationalisme naturalisé.

Par son idée de grammaire mentale universelle dont on peut décrire formellement les règles phylogenétiquement héritées. Noam Chomsky en fut un inspirateur si influent qu il est tenu pour responsable de la révolution en sciences cognitives contre l'associationnisme de Locke et de Hume, le behaviourisme de Pavlov et le constructivisme de Piaget. Chomsky a fourni à la psychologie cognitive son fondement: l'idée qu'une investigation scientificue permet de comprendre comment l'esprit se représente le monde et quelles règles il suit pour manipuler ses représentations. Ces règles sont innées, ne pouvant être dérivées de l'information, trop pauvre, fournie par un stimulus sensoriel, et néanmoins formelles ou syntaxiques, c'est-à-dire indépendantes de et préalables a toute signification.

Jerry Fodor a fortement relayé les thèses de Chomsky. Il a soutenu un nativisme radical qu il oppose au behaviourisme et à la sélection darwinienne. Pour lui les stractures du langage mental (le mentalais) sont innées comme l'est la syntaxe de la grammaire universelle. L hypothèse du mentalais se place dans une perspective physicaliste: les représentations mentales sont identifiables à des états neuraux et fonctionnent selon une syntaxe similaire à celle d"une grammaire générative. Cette thèse, notamment défendue par Zenon Pylyshyn, est généralement rejetée par les psychologues du développement, qui ont une position plus nuancée, acceptent la sélection naturelle et limitent l'inné aux dispositions qui formeraient le point de départ du développement. Ils admettent en effet lexistence dun «noyau cognitif» (core cogmition). expression proposée par Elizabeth Spelke dès les années 1980 pour désigner un ensemble de prédispositions cognitives neuralement incaunées et psychologiquement vérifiées qui constituerait le point de départ du développement de la compétence numérique ${ }^{6}$ Les tenants de la « theory theony » supposent même que les enfants naissent avec des théories initiales qu'ils testent et révisent avec les mêmes mécanismes cognitifs et les mêmes raisonnements que ceux des scientifiques, usant en particulier de l'explication causale." Cependant des auteurs comme George Lakoff ou. Rafael Núñez contestent le nativisme, soulignent le rôle de la répétition de l'expérience et de la culture, et proposent une analyse top-down des capacités cognitives en prêtant une fonction centrale aux métaphores conceptuelles. ${ }^{*} D^{\prime}$ autres comme Roger Lécuyer font observer que l'expérience et l'interaction avec l'environnement commence dès le stade foutal." A quoi les nativistes répondent qu'ils pensent en termes d'un dialogue entre nativisme et empirisme psychosociologique. ${ }^{\text {In }}$

Je n'ai pas l'intention d'entrer dans le débat sur l'inné et l'acquis. Et cela

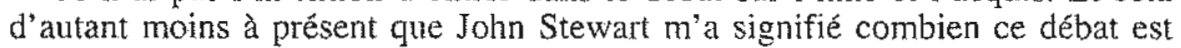
constamment mal engagé : sont rarement précisées les conditions de validité d"une triparfition entre variations génétiques, variations environnementales, et interactions statistiques entre facteurs génétiques et facteurs environnementaux, cette tripartition n'ayant de pertinence que dans le cas ou un caractère est variable dans une population donmée. De plus, on gagne certainement à laisser tomber l'opposition rigide entre inné et acquis, et peut-être aussi ces concepts eux-mêmes, même une fois rendus flexibles."

Cependant, penser selon des couples de concepts antagonistes parait irrésistible. La dialectique du mêrme et de l'autre est aussi ancienne que la philosophie de Platon. Et des anthropologues comme Claude Lévi-Strauss ou Françoise Héritier ont montré la généralité de la pensée par reiations binaires d'opposés, comme le cru et le cuit, le chand et le froid, le sec et l'humide. le dur et le mou, le haut et le bas, le dedans et le dehors, l'actif et le passif, le

"(Speke, 1983, p. 29): «[...] adils have a core conception of objects, and it is the very conception with which infants are bown. Development brings us new conceptions, some of them quite general, bur these new conceptions do not displace the core cognition. New conceptions of objects grow on the periphery of a set of notions that are in hate, that are present troughtoul life, and that do nof change" ${ }^{7} \mathrm{Par}$ thcorie il caut entendre un ensemble de representations abstraites, complexes et hautement structurées. Voir (Gopnik, 2003).

* where York. Basic Barks, 2000. Une "métaphore conceptuelle" est un outti] de transfort de relations du domaine des objets ou actions physiques au donaine arithmetique, permettant ainsi de construire des concepts et des relations abstraitcs. Du groupe d'objets physiques on passe à l'addlition ef à la multiplication des nombres entiers. On peut mêne voir la commutativité de lit multijlication $2 \times 3$ sur le rectangle de largeur 2 et de longueur 3 en faisant faire au rectangle un quart de tour : cela ne nécessite ni le compiage ni mêne le savoir que le gésulint est égal à 6.

${ }^{9}$ On trouvera un examen récen! du débat entle nativisme et constuctivisme signé par (Lécuyer \& Durand, 2012).

"Voir par exenaple, (Spelke, 1998), De son côté Fei $X_{u}$ emvisage une approche

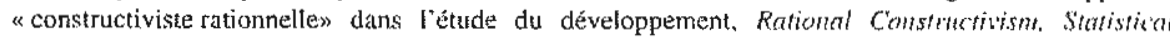
Enference, culd Core Cognition, in (Carey Z011, p. 151).

"(Stewart, 1993) critique l'isage du couple de concepts d'inné et d'acquis et fait très justement remarquer que l"information nécescaire à la réalisation d'un processus biologiquese at a fortion psychologique, ne lui préexiste pas mais est dynamigueetment constituée par le processus lui-mêne de manière contingente et opportumiste (p. 11 et sq.). 
masculin et le féminin, sans parler du couple nature/culture." Qu'il puisse "agit de constructions sociales conventionnelles plus ou moins récentes ou d'oppositions abstraites ne reflétant aucune discontinuité réelle n'empêche pas leur effet de structuration de notre appréhension actuelle et située du monde. Mais la dualité des catégories de pensée n'a pas à se muer en dualismes philosophiques posant plus de problèmes qu'ils n'en tésolvent en concevant des pôles de réalité sêparés, in éductibles et hiérarchisés.

Revenons à l'expérimentation neurobiologique et psychologique. Outre un partage entre inné et acquis, les tests conduits par neurophysiologistes et psychologues du développement et impliquant des quantitês ou des ensembles d'obiets supposent ou induisent des options relatives aux rapports entre évaluation de la guantité et comptage, entre continu et discret, entre aspect ordinal et aspect cardinal du nombre, entre perception sensorielle et concept, entre perception qualitative et perception quantitative, entre intuition et inférence, entre image et pensée, entre pensée et langage. La détermination de ces rapports dans le sens d"une antériorité ontologique ou cognitive de l'un ou Fautre terme de ces couples. par exemple du continu sur le discret, de la qualité sur la quantité et de la quantité sur le comptage, dépend non seulement de l'âge des sujets testés, des types de questions posées, des techniques d'investigation privilégiées et de leurs mises en ceuvre variées, mais aussi des modèles mathématiques de représentation des données et des options philosophiques déclarées ou tacites qui encadrent l'interprétation des réponses. Outre le fait troublant qu“il n'est pas rare que certains résultats d'expériences en contredisent d'autres, beaucoup de résultats empiriquement incontestés se prêtent à des interprétations différentes, ce qui jette un doute sur la signification thêrique des faits dexpérience. De fait. le catactère spéculatif des hypothèses avancées pour expliqter les résultats expérimentaux est souvent reconnu par leurs auteurs mèmes ${ }^{13}$.

Il $n$ "en reste pas moins que certains expérimentateurs utilisent plus ou moins explicitement un quadrilage de leurs résultats en les distribuant par rapport a un ou plusieurs de ces couples de concepts, en opposant par exemple une perception sensorielle, qualitative, continue et imagée à une approche discrète, numérique, et conceptuelle ou langagière (Stanislas Dehaene) D'autres ne mettent pas en avant un tel quadrillage ou en déplacent les démarcations. en particulier lorsqu ils ciblent les processus génériques de catégorisation, dont la catégorisation quantitative et numérique n'est qu'une espèce. certes fondamentale (Zenon Pylyshyn. Susan Carey).

\footnotetext{
"Générabité ne veut évidemmant pas dire usiversilisé rondamentate a incontournathes. Selon Philippe Descola la distinction entre mature et culturt est une acquisition accidentule récente (Par-delà nature et chlure. Gallimard, 2005).

"Par exenple (Speclke. 1498. p. 190-191): "Like any other branch of science, He study of cogninge

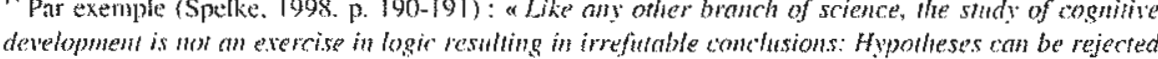

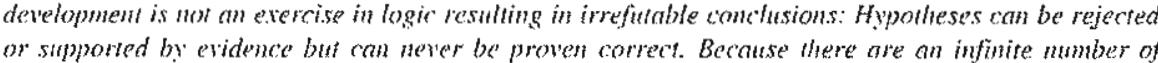

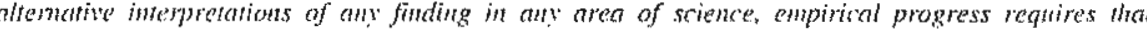

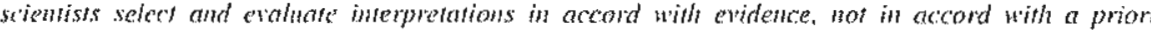

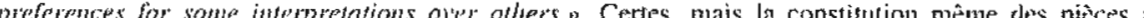
conviction tbëi néctssairénent quoique relativenent à des idées directrices.
}

\section{II - LE SENS DU NOMBRE}

\section{Il.1 - Biologie et cognition}

Les investigations neurobiologiques ont mis en évidence l'existence d'une saisie directe et immédiate de la quantité, sans effort conscient et indépendamment du langage et du raisonnement. Les expériences sur des animaux (par exemple les rats de Mechner, 1958 ou de Platt \& Johnson, 1971 ; les singes de Niecier \& Miller, 2003, 2004) et avec des peuples ne disposant pas de mots pour des nombres supérieurs à 5 (les Mundunkús d'Amazonie étudiés par Pica et al., 2004, Pica, Dehaene et al., 2013 et les Pirahãs étudiés par Peter Gordon, 2004) confirment les données recueillies avec de jeunes nourrissons ${ }^{14}$ en faveur de l'hypothèse d'une saisie directe et indépendante du langage. Ce que les nourrissons et certains animat $x$ saisissent directement c'est la numérosité d'un groupe d'objets, par contraste avec le nombre de ces objets, lequel résulte d'un dénombrement ou d'un comptage précis. Aucun comptage n'est en effet impliqué dans l'appréciation rapide de la taille d'un grand nombre d'objets, ni même dans la saisie directe du nombre exact d'objets lorsque ce nombre est inférieur à quatre. Le nombre est culturel tandis que la numérosité serait une donnée naturelle ou primitive.

De nombreux auteurs en effet voient en la numérosité le précurseur du nombre, et en attribuent la saisie à un sixième sens, comparable aux sens de la vue, du toucher, de l'oule, de l'odorat et du gout : le sens du nombre. Il s'agit, écrit Stanislas Dehaene, d'un "véritable sixième sens, quit permet de percevoir le nombre au même titre que la couleur, la forme ou la position des objets, et offre a l'animal comme à l'homme un instinct du nombre, une intuition directe des quantités numériques ${ }^{15}$ Le sens du nombre détecte la numérosité avant tout processus de comptage. L'expression 'le sens du nombre', empruntée a Tobias Dantzig's et populatisée par Stanislas Dehaene, surtout par le titre de son livre en anglais The Number Sense. How the Mind Creates Mathematics", a fait florès. Elle n'est pourtant pas à prendre à la lettre. En 2001 Dehaene publie un Précis of The Number Sense's où il la présente comme une métaphore" signifiant une appréhension rapide et immédiate de la quantité et une discrimination globale et approximative entre quantités différentes. La métaphore est largement usitée par neurobiologistes et psychologues du

${ }^{14}$ (Xu \& Spelke, 2000).

is (Dehaene, 2010 , p. 9 ).

"Number: The Langtrage of Science, Macmillan Company, 1954

"Oxford University Press, 1997. Conune La basse des maths la deuxiène édition de The uumber Sense. How the Mind Creates Mahlhematics, pubilée en 2011, comporte un nouteau et demier chapitre qui tient compte des rucherches postérieutus à 1997 et rcmanie parfois de manière importante, les conclusions défendues clans la première édition.

${ }_{18}$ (Dehaene, 2001). Voir également les six cours donnés aa Collegte de France en 2008

https:/titunes.apple com/fr/tunes-u/psychologie-cognitive-experimentale $/$ id 429645959 ?mt=10.

(hitp://citeseerx.ist.psu.edu/viewdoc/townload?doi=10.1.1.4.1207\&rcp=repl\&type=pdf) et le résume substantiel publie dans f'Annuaire du Collège de France $n^{\circ}$ 108, 2007-2008, Psychologie cognitive expérimentale, p. 277-301. URL : http://tanuaire-cdf, revues.org/1/4.

${ }^{19}$ "Number sense is a short-hand for our ability to quickly understand, approximate, and manipulate mumerical quantities." 
développement pour désigner l'accès perceptif primaire à la numérosité Lhypothèse propre à Dehaene consiste à avancer que les circuits cérébraux activés par une perception ou une manipulation élémentaire de la quantité (comparaison, addition et soustraction élémentaires) sont spécifiques et que cette spécialisation neuronale est le produit de l'évolution biologique selon les principes de la sélection darwinienne."

«Les mêmes pressions sélectives qui ont façonné le délicat mécanisme de l'ceil, le profil de l'aile du colibri, ou ta minuscule robotique de la fourmi. se sont également appliqués au cerveau. Au fil des ans et des espèces des organes mentaux toujours plus spécialisés: s'y sont épanouis afin de mieux exploiter l'afflux d'informations sensorielles qui lui parvenaient, et donc d'adapter l'organisme à un environtement parvetalent. et donc d aulap

Pour Dehaene l'aptitude numérique est biologiquement déterminée, ce que pensent également de nombretix scientifiques qui ne partagent pas intégralement tous les traits par lesquels Dehaene décrit cette aptitude tenue pour innée. En particulier il n'est pas universellement admis que l'ontogénèse reflète la phylogenèse. c'est-à-dire que le développement individuel reproduit l"évolution des espèces. Même si le pré-câblage ne fait pas de doute, on peut défendre l'idée que nos aptitudes numériques ne sont pas de simples domées de l'évolution : la psychologie du développement met en évidence les chemins qui du pré-câbląge mènent à la connaissance arithmétique et relativise ainsi, sans le nier, le caractère inné de l'aptitude numérique observable dès les premieres heures de vie. Certains plaident pour la recherche de fondements propsement cognififfs distincts des fondements cérébraur ${ }^{2 *}$ de l"arithmétique et invitent à une perspective constructive plus générale, le sens du nombre n'y étant présenté ni comme l'unique ni comme le tout premier outil de la compréhension et de la construction des nombres entiers (S. Carey). D'antres pensent que les mécanismes de représentation numérique ne sont pas génétiquement déterminés mais historiquement et culturellement formatés et se concrétisent dans la variation phénotypique du cerveau humain ( $R$. Núñez").

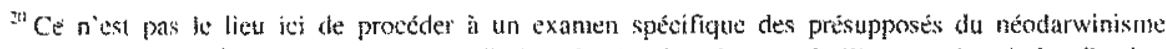

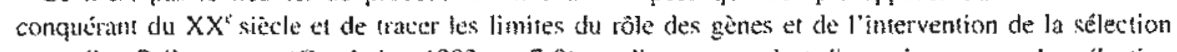
naturefts. Brièvement. (Goodwin. 1993. p. 7-8) soulignte que c'est l'organisme. non la sélection maturelje. çui est « un agent actif avec ses propres principes d'organisation. interposé entre les gènes et l'environnernent n. Poar Goodwin « une deseription de l'śvolutiont plus pettinente que celle foumie pas

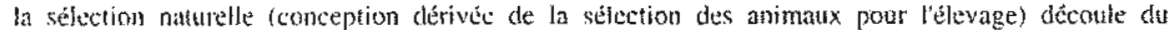
concept de stabilité dynamique ». Plus perrinente et plus fine en effet.

$\Rightarrow$ Annuaire du College de France, 2007-2008. p. JII : voir aussi (Dehaene. 2010. p. 270-277): a les numbres sont des comstructions mentales dont les racines ptongent. en derwière analyse, dans ladapiation fó cerveau humban aux régularilés de l'univers w.

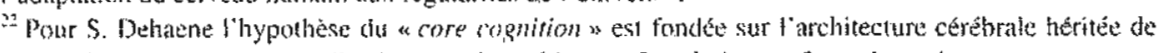

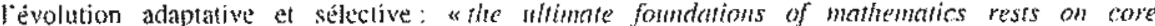

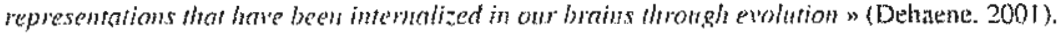

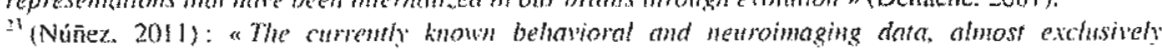

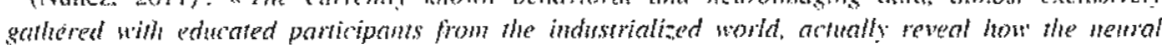

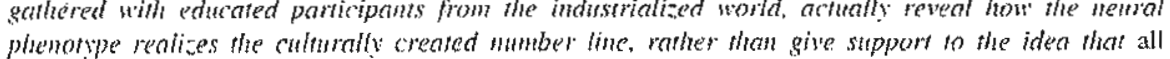
Hono Sapiens individnals map numbers to space in a menter line mommer n.
}

Je vais m'intéresser essentiellement ici aux discussions sur les rapports entre aptitudes proprement numériques et aptitudes conceptuelles plus générales en vue de discerner ce qui relève spécifiquement du nombre au sein des processus de catégorisation. Je m'appuie sur un ensemble largement non exhaustif de publications de spécialistes et je me sers principalement de trois tivres, qui me semblent paradigmatiques dans leurs perspectives respectives en ce que 1) ils rassemblent de très nombreuses données recueillies par différents chercheurs de différents laboratoires dans des expériences diverses par leurs technigues, leurs protocoles, leurs objectifs particuliers et 2) ils présentent ces données dans le cadre de thèmes ou visions philosophiques explicitement précisées. II s'agit des livres de Pylyshyn (2002, Seeing and Visualizing ${ }^{24}$, de celui Dehaene (2010 ou 2011) et de celui de Susan Carey (2009, The Origin of Concepts).

\section{II.2 - Les cartes du cerveau}

Commençons par rapporter les résultats de l'imagerie fonctionnelle par résonnance magnétique nucléaire, qui fournissent des données neurophysiques matériellement présentées: les images montrent l'activation de circuits de neurones lors de tests des aptitudes numériques. Interpréter ce que l'on voit peut certes ne pas conduire à des conclusions totalement concordantes, mais un consensus s'établit plus facilement sur la topographie cérébrale que sur la signification des données psychologiques. Que montrent donc les images du cerveau?

Selon Dehaene et les neurobiologistes le sens du nombre est matériellement traduit par l'organisation topographique d'une région spécifique du cortex du lobe pariétal des deux hémisphères. Cette organisation est décrite par un ensembie de données qui sont essentiellement les suivantes:

1) la région bilatérale des flancs du sillon intrapariétal est spécifiquement activée quand il s'agit de comparer des petites quantités comme let 2 ou 2 et 3 ou d'estimer approximativement une quantité plus grande que 4 . Cețe région occupe une position bien précise au sein d'une mosaïque de régions sensori-motrices impliquées dans les mouvements des yeux, de la main ou du doigt (Simon, Mangin, Cohen, Le Bihan, \& Dehaene, 2002).

2) Les expériences sur des singes ont montré qu'il existe des populations de neurones préférentiellement dédiées: certaines populations sont activées par la présentation d'un objet unique, d'autres par la présentation de deux objets, d'autres par la présentation de trois objets, quatre objets, cinq objets (Nieder, 2005), et ainsi de suite jusqu' a 20 ou 30 objets (Nieder \& Merten, 2007). Plus exactement chaque population de neurones répond à un pourcentage de nombres autour de sa valeur favorite, obéissant ainsi à une courbe en cloche caractéristique de l'estimation approximative. La taille, la couleur, la disposition spatiale des objets n'entrainent pas de changement de ces «neurones des

\footnotetext{
${ }^{24}$ intp://ruccs. rutgers.edu/faculty/pylyshyn/bookall.pdf.2002.
} 
nombres", qui sont localisés dans - ou à proximité immédiate de ]'aite ventrale intrapariétale (VIP), dans les profondeuts du sillon intrapariétal.

3) Un autre type de code neurä de la numérosité existerait chez les singes dans une région pariétale pius latérale et postérieure, l'aire LIP (Roitman. Brannon \& Platt, 2007). Les neurones de l'aire LIP ne sont pas accordés à un nombre préféré, mais leur taux de décharge varie en fonction monotone, croissant ou décroissant avec le logarithme de la numérosité de l'ensemble présenté et obéissant ainsi à la ló de Weber-Fechner. ${ }^{25}$ Il semble que l'extraction initiale de l'information se fait en deux étapes, dans l'aire LIP, puis dans laire VIP, tandis que sa mémorisation impliquetait préférentiellement le cortex préfrontal dorsolatéral

4) Liactivation de la région pariétale inférieure n'est pas affectée par le mode de présentation de la quantité, par exemple sous forme de nuages de points ou sous forme de symboles arithmétiques.

5) Dès les premiers mois de vie des êtres humains il existe deux voies distinctes pour le traitement de la forme et du nombre: cortex visuel central d'un côté, cortex pariétal de l'autre (Izard, DehaeneLambertz \& Dehaene, 2008). Ce résultat affine celui rapporté en 1$)$.

6) La saisie de la quantité est abstraite en ce sens précis qu“elle met en relation des informations provenant de différents organes sensoriels : des bébés de denx jours accordent le nombre de sons entendus au nombre de points vus sur une image ; mais cela ne veut pas dire que le nombre est, à ce stade, dissocié de son support sonore ou visuel ${ }^{17}$; à ce stade il n'est donc pas «abstrait » au sens usuel de ce terme hérité d'Aristote ${ }^{2 *}$

7) La durée et l'intensité d'activation de la région pariétale inférieure sont dépendantes de leffet distance (on distingue plus facilement entre 80 et 100 qu'entre 80 et 82 ).

8) Les circuits activés par la comparaison et l'estimation approximative sont différents de ceux activés par un calcul exact: seuls ces derniers impliquent des régions spécifiques du langage. Certaines lésions en effet entrainent la perte de capacité de lire des noms de nombres sans que la capacité de comparaison et d'estimation approximative des quantites soit affectée.

\footnotetext{
${ }^{2}$ Celle-ci powe que la sentistlion varie comme le logarithme du stimulus. La plus petite difference perçue entre deax stimulations est proportionnelle à l'intensité du stimulus maltiplié par une constante $k$ dite fratetion de Weber.

2" (Starkey. Spelke \& Gutman. 1990 ; Izara. Sann. Spelke. \& Streri. 2009).

$\Rightarrow$ (Feigenson \& Spelke. 1998 : Lécuyer. 2002 )

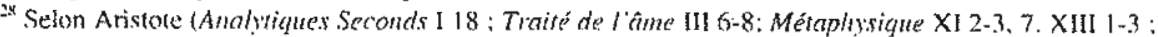
Physique' 11. 2). l'abstrait c'est le sépauré par soustraction (apoipeors) d'aspects considérés comme accidentels ol changeants. Cette operation de soustraction est selon Aristole le mode de formation accidentejls ou changeants. Cette operation de soustraction est selon
logique des concepts. grâte auxquels on passe du particulier au général.
}

9) Associer une quantité à un symbole, par exemple deux points au chiffre 2, exige au début le recrutement des neurones du cortex préfrontal, le « cortex de l'association 》 (Diester \& Nieder, 2007), qui a une fonction intégratrice, en particulier des neurones de la région temporale inférieure qui reconnaît les formes vistaelles et de ceux de la tégion pariétale qui code les quantités. Le cortex préfrontal est aussi la région qui permet de conserver l'information en mémoire. Une fois l'association établie l'activation se déplace vers les régions partétales et occipito-temporales. Chez l'aduite éduqué c'est bien la légion intra-pariétale, et plus précisément le segment horizontal du sillon intra-pariétal (hIPS), qui est le lien de rencontre des quantités et des symboles. Ce résultat précise celui rappotté en 4 ).

10) De récentes expériences montrent que la spécialisation numérique du lobe pariétal droit est antérieure à l'acquisition du langage ${ }^{30}$ Au cours de l'éducation c'est surtout la région pariétale gauche qui est modifiée, tant dans son activité de base que dans la taille de son effet de distance, ce qui suggère un affinement de la précision du codage des quantités au sein de l'hémisphère gauche (Ansari \& Dhital, 2006). L'apprentissage engage en effet les aires du langage et de la mémoire, tandis que les neurones pariétaux des quantités concrètement présentées sont recyclés en neurones de symboles mumériques..$^{30}$ II apparaît donc que la région pariétale gauche abrite un code des quantités et les connexions directes pour y accéder à partir des aires linguistique et symbolique de l'hémisphère gauche. L'imagerie par résonnance magnétique fonctionnelle montre qu'un ensemble concret d'objets active une assez vaste région de neurones pariétaux, tandis qu'un nombre présenté par le biais d'un chiffre arabe induit une activation plus focalisée sur un sousgroupe de neurones pliıs sélectifs.

En bref, d'un côté le segment horizontal du sillon intrapariétal (hIPS) est. spécifiquenent dédié à une appréhension de la quantité dans une dimension abstraite au sens où elle est amodale, c'est-à-dire indépendante du mode de présentation de la quantité : visuel (nuage de points, ou chiffres arabes ou noms de nombres "un', "deux", etc.) ou auditif (suite de sons) et indépendante des paramètres de taille, de forme, de couleur des éléments présentés. À la différence donc des organes des sens, spécialisés chacun dans une modalité sensorielle, le sens du nombre traite des informations livrées par différents organes sensoriels. Il faut souligner que hIPS n'intervient pas dans la comparaison ou la classification de types d'objets autres que les quantités, par exemple lorsqu'il s'agit de dire si un animal est un mammifere ou non, ou lequel de deux animaux présentés en image est le plus féroce." La question se pose néanmoins de savoir si la numérosité engage une aptitude de catégorisation spécifiquement formatée pour elle.

\footnotetext{
${ }^{93}$ Par exemple (Hyde, Bons, Biair \& Carey. 2010).

${ }^{30}$ (Anderson, 2010)

${ }^{31}$ (Dehatene, 2010, p. 286: Dehaene. 2011, p. 243).
} 
D'un autre côté ancune partie de hIPS n'est dédiée exclusiventent à la quantité ou aux opérations arithmétiques élémentaires." II $n^{*} y$ a pas de " module numérique » au sens de Butterworth (1999). Il apparaît même que la grandeur, qu"elle soit numérique, spatiale, temporelle, lumineuse, active des régions qui se recouvrent tout au long du sillon intrapatiétal. En particulier la position des objets dans l'espace et leur taille interférent avec l'estimation de la quantité et de la grandeur : nous utilisons des circuits qui aident nos mains et nos yeux à s'orienter dans l"espace ; les notions concrètes de taille. d'espace et de temps sont impliquées dans lappréhension de la quantité en tant que quantitê. ${ }^{3}$. c'est-à-dire de la quantité dans une dimension abstraite, où «abstraite » ne se réduit pas ici à «amodale». Certains insistent sur l'enracinement sensoriel et neural de la saisie de la dimension abstraite des événements et des phénomènes qu'est leur grandeur ou leur quantité (Debaene). D'autres en concluent à l'intrication originelle de la perception sensorielle et de la catégorisation pré-conceptuelle préverbale : percevoir n'est pas recevoir passivement mais saisir activement, percevoir n'est pas seulement être impressionné mais aussi d'emblée filtrer catégoriquement ces impressions (Spelke, Carey). D'autres encore font la différence entre structuration perceptive et conceptualisation (Pylyshyn). Plusieurs positions oscillent entre I'idée que la signification numérique est directement donnée dans la perception et l"idée que la perception comporte une construction de la signification. Du poin de vue épistémologigue, il faut d'emblée souligner que les cortélations établies entre les circuits neuraux et les fonctions sensorielles, ou entre les circuits neuratx et les comportements cognitifs sont et restent des corrélations. non des explications au sens propre. Par elles-mênes les cartes du cerveau ne justifient pas le réductionnisme matérialiste et déterministe que l’on voudrait fonder stit elles.

On notera ici que la théorie constructiviste de Piaget, qui a servi de repoussoir à l'élaboration du nativisme. enseignait pourtant que la perception est déjà une activité de mise en relation. On observera aussi que, contrairement a ce qui se passe souvent avec certaines doctrines philosophiques réalistes qui prêtent aux nombres une existence extra-psychique, intuition d'un donné numérique (d'une numérosité) et construction du nombre ne sont pas opposées et apparaissent expérimentalement compatibles et complémentaires, donné et construit appartenant tous deux à la sphère neuro-psychique. ${ }^{3}$ Enfin on remarquera le rôle capital des zones de la perception, de l'attention et de la

B(Dehatene, 2010. p. 285: Dehaene, 2011. p. 243)

"(Hubbard. Piazza. Pinel \& Dehaene, 200s) : a [.. I menterical-spatial interactions ran far teeper than

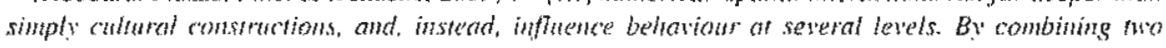

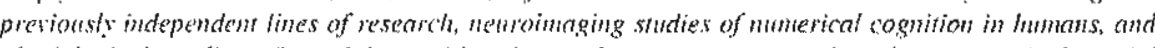

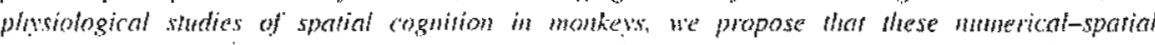

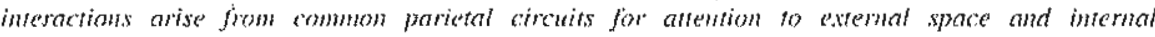
replesetiations of numbers. "

Une confirmation récente est apportéc pat (Spelke. Jzard. Couthart. de Hevia \& Streri. 2014):

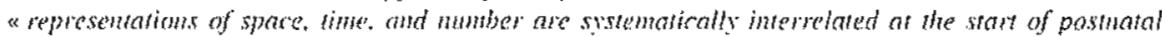

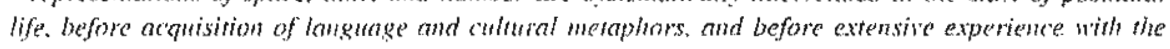
natural currelations betnitent the se dinnensions.

${ }^{3}$ Pour Dehnente. "l'inturition, Join d'êlre inaccessible à l'étude scientifique. possëde une signature gaychologique el netrale déchiłfrable ». Annuaire du Coltège de France $2007-2008$ mémoire de travail dans l'appréhension et la manipulation des nombres : par exemple, ce sont ces zones qui sont principalement activées chez les calculateurs prodiges. Daniel Tammett témoigne en effet qu'il voit littéralement les décimales de $\pi$ défiler devant ses yeux comme les images d'un film et que si on lui demande d'additionner ou de multiplier deux nombres il voit mentalement les nombies proposés et le résultat s'offre visuellement à son esprit sans qu'il ait eu besoin de le calculer analytiquement. ${ }^{35}$

La gégraphie du cerveau n'a pas fini d'exciter la curiosité des neurobiologistes. La revue Science vient encore récemment de publier une étuđe confirmant plusieurs données sur la topographie cérébrale qui justifient l'hypothèse d'un sens du nombre. Les auteurs pensent que la saisie de la numérosité est l'affaire de régions incluant le cortex préfrontal et le pariétal postérieur, mais que certains aspects de la saisie « reflètent * (mirror) les processus de la perception sensorielle et motrice à laquelle correspondent notoirement des cartes cérébrales. On peut en effet pour chaque sens dresser une carte cérébrale correspondant à la structure et an fonctionnement de l'organe sensoriel en question; par exemple le cortex visuel est divisé en aires régissant respectivernent la forme, la couleur, le mouvement, etc. L'organisation cérébrale topographique des circuits de neurones accordés aux numérosités est analogue ; par exemple une plus grande surface corticale est engagée dans la saisie des petites numérosités que dans celle des grandes numérosités et la surface est plus grande pour la numérosité préférée. La saisie de la numérosité ressemble à une perception sensorielle; le sens du nombre est comme un sixième sens : l'expression est plus qu'une métaphore, elle désione une capacité biologique à saisir une propriété abstraite d'une manière neuralement analogue à la perception sensori-motrice. On en conclut à un enracinement neural concret d'une prédisposition biologique à l'abstrait ainsi que le soulignent les neurobiologistes." Enoncée sous cette forme générale cette conclusion (que je tire de l'article en question) n'implique pas encore que cette prédisposition biologique a l'abstrait se traduit par l'image mentale d'une ligne continue orientée. Du reste, les auteurs de l'article notent que le chevauchement des représentations vistuo-spatiales et quantitatives peut sans doute sous-tendre l'image de la ligne numérique mentale (LNM) si fortement défendue par Dehaene ${ }^{70}$ ou Gallistel et Gelman", bien que les réponses visuo-

"Voir l'article "A Genius Explains". in The Gurdion, 12 fevrer 2005. Quand D. Tammet multiplie deux nombres, « il voit deux fornes, pulis l'innage commence à changer ex une troisične forme émerge. C'est la response. Il s'agit d'imagerie mentale. C'est comme faire des maths sans penser, o Pliss exactenent e'est penser visucllement (visual shinking). Cependant des réserves sont formulées à l'encontre de la similitude entre image mentale et vision, par exemple par Pylyshyn (2003. p. 115 ): " cortical images occur in both vision and inagern, the difference being that the former is coused by Iight on the retina white the latter is caused by top-donm projections from higher cognitive systems, "s ${ }^{\text {th }}$ (Harvey, Klein, Peridale \& Dumoulin, 2n+3)

${ }^{3}$ Une confirmation sera apportée plus bas dans l'article, quand $j$ 'analyserai les mécanismes de saisie catégoriale des objets.

${ }^{3}$ Cf. par exemple (Dehatne, 2001, p. 3) : « My hypothesis is that the number sense quralifies as a biologically determined categon of knowledge. I propose that he fourdarions of arithmetic lie in our ability to menally represent and maniputate numerosites on a menal "umber tine", an analogica representation of number; and that this representation has a long evolutionary history and a spectife cerebral substrate. » 
spatiales et les réponses quantitatives aux tests proposés de dénombrement sans comptage ne montrent pas de relation consistante entre les deux ensembles de réponses. Autrement dit, le modèle d'appréhension analogique de la numérosité proposé par Dehaene comme correspondant à un des systèmes fondamentaux de la cognition numérique-ANS pour analogical number system - n'est pas confirmé expérimentalement d'une façon absolùe. Du reste. certains en contestent le caractère inné. R. Núñez par exemple fait observer que ce modèle résulte d"un acquis culturel relativement récent de la civilisation européenne. La coordination espace-nombre semble n'apparaitre qu'au XVII siècle dans le Treatise of Algebra (1685) du mathématicien John Wallis. Ni les tabletles de Mésopotamie ni même La Géométrie (1637) de Descartes ne comportent de tracé de ligne numérique." D'autres auteurs proposent des modèles d'explication alternatifs qui rendent compte différemment du rapport dı nombre à l'objet (Pylyshyn) et à l'espace physique perçu (Speike. Carey). Jy reviens plus bas. Mais d'abord dressons un bref répertoire des opérations mentales impliquées dans l'appréhension et la construction du nombre

\section{III - LES OPÉRATIONS MENTALES DU TRAITEMENT DE LA QUANTITÉ}

Les opérations mentales sont les médjateurs entre l'environnement et le comportement. D'après la plupart des experts en sciences cognitives (Dehaene, $\mathrm{Xu} \&$ Spelke. Wynn. Carey. etc.) on distingue quatre types dopérations traitant de la quantité et correspondant à des circuits neuronaux dissociables.

1) L'appréhension subite et directe de la quantité, dite subitisation, concerne un nombre d'objets limité au mieux à 4 , à 3 pour les nourrissons ou même 2 pour les tout nouveaux nés. Dehaene et Cohen ont montré que les patients simultanagnosiques peuvent encore subitiser mais pas compter, ni résoudre des calculs simples: incapables de dire par exemple si $2+2=5$, ils peuvent dire que $2+2$ n"est pas égal à 9." Une première hypothèse défendue par Dehaene consiste à penser que ta subitisation a lieu sans effort d'attention sériellement porté sur chacun des objets tour à tour. Mais on a pu montrer que si la subitisation n'exige pas d'effort conscient, elle mobilise l'attention et la mémoire de travail. Si elle est bien un élément deı noyas primitif de la cognition numérique, alors il faut modifier la caractérisation de ce noyau primitif en y incluant l'attention préverbale et la mémoire. ${ }^{+2}$ Dehaene en convient. ${ }^{.2}$

${ }^{3}$ (Gallistel \& (ielman. 2000) argutun que notre machinerie cérébrale primilive travaille avec les nombres réels avam d'en venir itux nombres entiers et (Gallistel \& Gelman, 2006) parlent de l'existence matériellement incarnée dans Je cerveat d un systène de monbres réts.

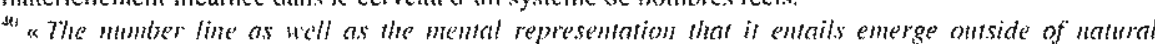

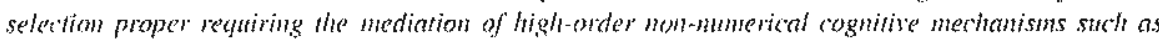

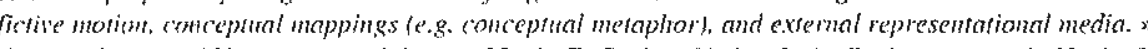
(Núnez. 2011. p. 652) Lanticle cité. note 33, de E. Spełke. V. Lzard. A. Coubart. M.D. de Hevia \&

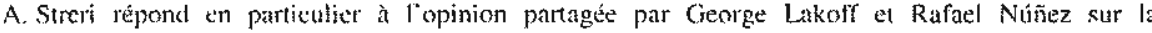
consunction cullurelle des concepts mathematiques

+1 (Dehare \& Colren. 1991)

H: $(X u . F .2($ F $) 1)$
2) L'estimation approximative de la quantité concerne des quantités d'objets éventuellement beaucoup plus grandes: les nourissons distinguent entre un objet ef trois objets, mais aussi entre par exemple huit objets et seize objets. Quatre, ou peut-être déjà trois, est un seuil : tes nourrissons de cinq à six mois ne distinguent pas entre un objet et quatre objets, ni entre deux objets et quatre objets; les tout nouveaux nés ne distinguent pas entre deux objets et trois objets. ${ }^{44}$ L'estimation des grandes numérosités obéit à la loi de Weber-Fechner: l'imprécision de l'estimation crôt linéairement avec le nombre estimé, selon une constante de proportionnalité appelée coefficient de variation ou fraction de Weber; c'est pourquoi par exemple les nourrissons de six mois, qui distinguent entre huit et seize points, ne distinguent pas entre huit points et douze points ${ }^{+5}$, ils en deviennent capables à dix mois, ce qui atteste un accroissement rapide de la précision dans l'estimation d'ensembles ayant plus de quatre objets.

La subitisation et l'estimation approximative étant disponibles dès les premiers jours de vie des humains, on a pu penser qu'elles impliquaient un seul et même mécanisme inné d'accès à la numérosité, tandis que le nombre proprement dit est ensuite établí par comptage et par calcul. C'est la position de Dehaene en 1997 Mais, à la suite de nouvelles recherches, dont certaines menées dans son laboratoire par Manuela Piazza en 2003 et par Susannah Revkin en 2008, Dehaene est conduit à distinguer entre subitisation, appréciation exacte mais restreinte à trois objets au plus, et estimation approximative d'objets dont le nombre dépasse trois ou qtetatre : seraient donc présents chez le jeune enfant deux systèmes distincts dont l'intégration se fait plus tard, entre trois et quatre ans. Reste à savoir s'ils sont spécifiques au nême degré du sens du nombre. L'opinion de Dehaene et de certains autres spécialistes continue d'obéir à une priorité du continu sur le discret. Dehaene soutient en effet que «du système d'approximation vient notre intuition immédiate des nombres, tandis que le système des petits nombres, lui, apporte la possibilité de zoomer sur le comportement particulier des nombres 1,2 , et 3 et de comprendre leur arithmétique exacte $\gg$. Cela signifierait-il que nous n'avons pas d'intuition immédiate d'un ensemble discret de un, deux, ou trois objets et que lintuition immediate est the saisje

\footnotetext{
${ }^{\mathrm{N} 3}$ (Feigenson, Dehaene. \& Spelke, 2004): « Two distinct core systems of numerical reprerentaions are present in human infauts and in other animat species, and the refore do not energe through individual

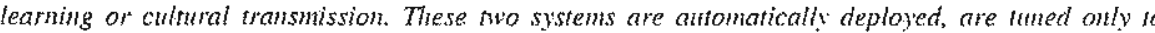
specific sypes of information, and continue to finction throughout the lifespat... The pwo systems $[\ldots]$ accoint for htumans' basic 'number sense' '. Même constat par (Revkin. Piazza, lzard, Cohen \& Dehaene, 2008, p. 607-614). Selon (Dehaene, 2010. p. 304-305; 2011, p. 259-260) : «e qui est singulier dars la subitisation e'est qu'elle foumit un chiffre discret... [tandis que] l'estimation approximative n'a rien pour supponter un systeme discrel de nombres ».
}

4 (Coubart, Izard. Spelke, Marie \& Streri, 2014).

*5 (Xu \& Spelke, 2000) 
nécessaitement qualitative et analogique de la quantité ? La question est toujours en débat et les opinions se partagent entre ceux qui pensent que le mécanisme d'appréciation approximative prédomine (Dehaene. Gallistel, Wynn) et qu'il est même possible de retrouver la loi de Weber dans la comparaison de petites numérosités sous certaines conditions dexpérimentation, pár exemple lorsque la tâche proposée entraîne une plus grande charge de la mémoire de travaift', cenx qui pensent que le mécanisme d'appréhension discrète relève d'un système plus général non spécifiquement dédié à la numérosité et qu'on ne peut établir d'équation entre le flou de l'intuition et la fluidité du contint (Pylyshyn. Carey), ceux qui pensent que chacun de ces deux mécanisnes est spécialisé l'un pour les grandes numérosités, l'autre pour les petites numérosités ( $\mathrm{Xu}$, Spelke, Hyde ${ }^{\mathrm{j}}$ ).

3) Le comptage est un moment crucial dans l'apprentissage arithmétique; il permet de dénombrer avec précision un ensemble quelconque. 1] consiste à apparier, un par un, chacun des objets présentés avec les éléments d"une liste de référence qui peut être non verbale (doigts. parties du corps). Ou verbale (noms de nombres). L'étude princeps est celle de Gelman et Gallistel (1978) qui établit une liste de cing principes censés guider le développement du comptage. ${ }^{\text {tx }}$ Le comptage est un processus sériel qui dépend évidemment de l'attention : il est acquis par les enfant entre deux et quatre ans et permet de disposer d"une représentation uniforme des nombres dont le sens est supposé antérieurement acquis par l'appréhension directe de la numérosité. Mais il semble que ce qui est acquis c'est le fait mumérique, la signification singulière de chaque nombre étant acquise progressivement." En effet. Yenfant comprend d'abord la signification de un, quelques mois plus tard celle de deux. puis de trois, puis de quatre. A ce stade l'enfant a compris comment déterminer exactement une quantité par comptage. Cependant, certains patients disposant de noms de nombres et sachant compter ne réussissent pas a déterminer si "neuf désigne une quantité supériente à celle désignée par "sepp" "s", ce qui tend à montrer le rồle spécifique d'une appreciation approximative de la quantite, entravee dans ce cas par la trop grande proximité des deux nombres (c'est l'effet distance).

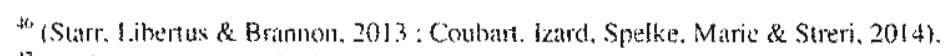

17 (Hyde \& Speike. $20099: 2011$ 1)

${ }^{45} \mathrm{Ce}$ sont: 1. We principe de correspontance un à un: chaque han énoncé kloit être mis en

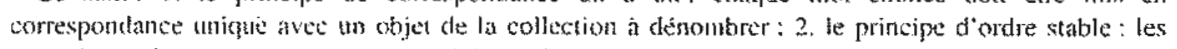

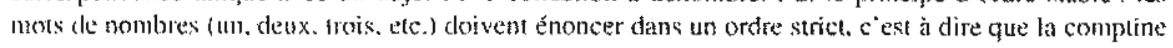

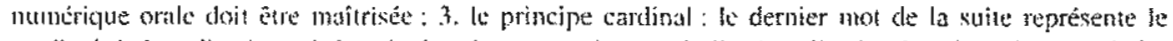
cardinal de la coltection: 4. le principe de non-pertinence de lardie : lortre dans lequel sont pris les

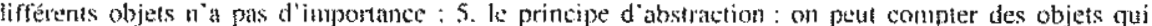
11 on pas de liens panticuliers entre eux. par exemple des lapins et des voitures présentés simultanément Sur un ecriln ous une imate.

"Wyon. 19y?).

41. (Dehatene \& Cohen. 1997).
Par ailleurs, anterrieurement au stade où il maîtrise la signification des nombres de un à quatre, l'enfant peut par exemple ne pas savoir compter jusqu'à trois et néanmoins faire la différence entre un et trois : or la différence entre trois et un est égale $a$ la différence entre neuf et sept, l'effet distance ne joue donc pas pour les nombres inférieurs à quatre. Pour ceux-ci la discrimination semble reposer sur l'existence implicite, préverbale et donc antérieure au comptage, de la relation de mise en correspondance un à un qui pemet une comparaison exacte. Certes l'ordre sériel n'est explicitement maîtrisé que par l'apprentissage progressif du comptage et la compréhension de l'engendrement uniforme de la suite des nombres par l'opération +1 n'intervient qu'aptès l'âge de trois-quatre ans, ce qui peut faire passer l'ordre pour un aspect secondaire du nombre par rapport à l'appréhension, la discrimination ou l'estimation immédiates de la numérosité cardinale. Pourtant la discrimination exacte entre petites numerosités, deux et trois par exemple. attestée par les expériences de Wynn sur l'addition ou la soustraction d'un objet (1992), peut incluire à supposer qu'il existe aussi une capacité innée à percevoir l'organisation sérielle d'objets.

4) Le calcul explicite au moyen de symboles numériques caractérise la compétence arithmétique. La capacité symbolique rétroagit sur les mécanismes d'estimation et de comptage en les renforçant et en les affutant. S. Dehaene développe l'hypothèse du « recyclage neuronal » d'Anderson: la plasticité cêrébrale bien reconnue permet de mobiliser les régions du lobe pariétal anciennement utilisées pour la reconnaissance des formes visuelles et des quantités et de les réorienter pour le calcul exact. Cependant de jeunes enfants continuent de recourir à des stratégies indirectes de comptage pour calculer symboliquement; par exemple pour exécuter l'addition 4+2. l'enfant va compter à partir de 1 jusqu'à 4 , puis ajouter un, puis encore un pour trouver 6 (dans certaines. conditions l'enfant ne trouve pas le rếsultat si on l'empêche de compter), C'est peu à peu que des processus mnésiques ou reconstructifs viennent supplanter la routine du comptage pour résoudre des opérations simples, l'enfant faisant appel à son stock de faits et de règles arithmétiques.

Les processus 1) et 2) sont présents chez les humains à leur naissance et chez de nombreuses espèces animales. Cela tout le monde l'accorde même si des discussions portent sur leur situation exacte sur P'échelle continue qui va des prédispositions innées aux constructions sophistiquées de la culture. C'est en eux, par opposition au comptage et au calcul symbolique, produits incontestes de la culture et de l'apprentissage, que l'on reconnaît le sens inné du nombte ou, pour être plus précis, de la numérosité. Contrairement à Gelman

5. Tandis que lá question du caractère inné ou hérité/versus aco ısis de l'aprifude numérique, peut êtro posée - mêne s'il s'avère que c'est une mauvaise question -, il ne fait pas de doule que la contpérence numérique est acquise par l'apprentissage et l'entraîneraent. 
\& Gallistel gui soutiennent que la subitisation est un comptage ultrarapide, la plupart des neuroscientifiques considèrent que la numérosité est obtenue par un dénombrement ou une évaluation sans comprage. Les processus qu'elle implique opèrent, selon Dehaene, par représentation et manipulation analogique du nombre selon une ligne mentale continue et orientée (de gauche à droite pour certains peuples. de droite à gauche pour d’autres) et compressée selon une échelle logarithmique. ce qui expliquerait l"imprécision plus grande de lestimation d'une grande quantité (effet taille) et la discrimination meilleure entre deux quantités dont l'écart est plus grand sur cette ligne (effet distance). La ligne mentale logarithmique de Dehaene correspond aux données électro-physiologiques de Nyder \& Miller (2003) et recueille une large adhésion parmi les psychologues dont les résultats expérimentaux attestent la présence đ"une estimation approximative des oumérosités supérieures à quatre dès les premiers jours de vie.s' Certaines expériences très récentes semblent même confirmer l'hypothèse d'un code analogique fonctionnant dès les premiers jours de vie pour la valeur cardinale de numérosités inférieures à quatre. ${ }^{\text {i }}$

Selon Dehaene la ligne numérique mentale (LNM) est censée exprimer la priorité cognitive de lappréhension directe et immédiate (par subitisation pour les petites quantités ou estimation approximative pour les plus grandes) de la numérosité par japport au comptage. cette priorité cognitive se conformant à une priorité ontologique du continu sur le discret et le sériel dans les phénomènes physiques, y compris ceux qui ont lieu dans le cerveau : s'il est difficile de comparer deux quantités peu écartées l'une de l'autre c'est en raison du recouvrement partiel des zones activées par l'une et l'autre, que ces quantités soient présentées par des nuages de points. des noms de nombres comme «un » ou «trois», ou par des chiffres comme « 1 ou « 3 ».

Il est admis que l"appréhension de la numérosité est le précurseur de la compréhension de la signification ${ }^{54}$ des nombres et de la mâtrise du calcul urithmétique. Mais l'yypothèse d'une homologie entre la continuité des phenomenes physiques contintıs (propagation du son et de la lumière, écoulement des fluides, etc.) el la continuité de notre perception vistuelle de la numérosité n'est pas universellement partagée. Zenon Pylyshyr et Susan Carey sinscrivent en faux tous deux mais de manière différente contre cette hypothesese: c'est un fait qu"un flux continu d"impressions sensorielles, visuelles par exemple, peut aboutir à la perception immédiate d'individus distincts que nous cherchons à atteindre ou entre lesquels nous établissons des relations spatiales: le contenu de la représentation mentale n'est pas le pur e simple reflet d'un stimulus sensoriel. La continutité numérique ne serait dono

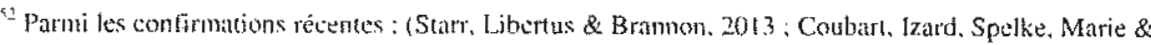

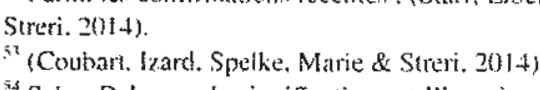

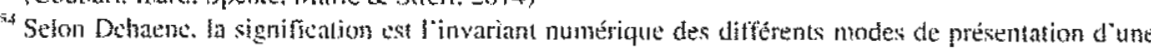

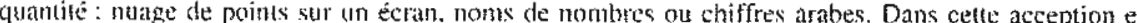

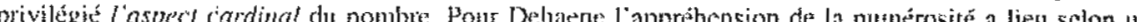
iriple code : lestimation analogique de ta vafeur cardinale. la représentation verbale de certe valeur par

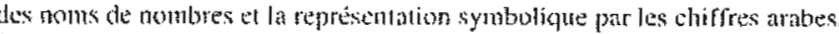

"(Pylyshyn, 201) 2: Carey \& Xu, 2001). pas le premier mais sellement un principe majeur de notre organisation du monde.

Avant d'envisager d'autres principes mis en évidence en particulier dans les travaux de Pylyshyn et de Susan Carey, je veux récapituler les questions philosophrques que suscitent les résultats livrés par la neuroimangerie et l'analyse du comportement en ce qui concerne le nombre.

1) La numérosité est-elle une propriété objective du monde et, si oui, est-elle une propriété primaire des phénomènes perçus? Ou bien est-elle un instrument d'organisation du monde utilisé concurremment avec d'autres outils par le sujet?

2) La quantité est-elle d'abord percue de manière quahitative avant d'être mesurée de manière exacte ou approximative, d'une manière analogue à la perception de la lumière blanche avant sa décomposition par un prisme en un spectre de couleurs distinctes ?

3) Y a-t-il une priorité ontologique et cognitive du continu sur le discret ou l'inverse?

4) L'aspect ordinal du nombre est-il secondaire par rapport à son aspect cardinal comme le pense Dehaene ou est-il déjà piésent dans la subitisation comme le pensent Gelman \& Gallistel, pour qui celle-ci est un comptage ultrarapide, et Susan Carey, pour qui la discrimination entre individus semblables mais distincts précède le comptage et l'estimation approximative?

5) La capacité très précoce de discrinination entre deux objets et trois objets est-elle une aptitude à distinguer des configurations différentes comme le soutient la Guestaltheorie ? Ou bien est-elle une capacité numérique de discrimination entre numérosités proprement dites ? Distinguer entre deux objets et trois objets implique-t-il de distinguer quantitativement entre deux et trois et de juger que trois est plus grand que deux ?

Je ne trancherai certes pas les difficultés inhérentes à ces questions dans les limites de cet atticle. Je vais privilégier un réseau de téflexions liées à l'ancestrale question épistémologique de savoir si les nombres (ou les formes géométriques comme le cercle et le triangle) sont des objets ou des concepts, c'est-â-dire des référents identitaires ou des réseaux variables de relations. Mes études sur les fondements de l'arithmétique et de la géométrie abstraites al $\mathrm{X}\left[\mathrm{X}^{\mathrm{e}}\right.$ siècle m'inclinent à la seconde option, constructiviste y compris pour ce qui concerne le point de vue formel. contre le réalisme de la première. ${ }^{50}$

${ }^{56}$ Une sétection de mes travaux apparât dans la bibliographie. Pour éclairer ma position, je signale. en particulier. qu' $x$ l'exemple de Cavailles, je comprends l'intuition non comme justiftention subjective d'un rénlismé des objets vus comme exterieurs à la pensée et revelés à elle. mais comme manfestation de l'autonomie acquise des toncepts mathématiques par mappor à la conscience. 


\section{IV - NOMBRE, OBJET ET CONCEPT}

\section{IV.I - Individuation parallèle}

La différenciation attestée expérimentalement entre la subitisation, restreinte aux petites numérosités, et l'estimation approximative conduit à repérer dans la première des processus absents de la seconde et moins facilement repérables au niveas neural par imagerie magnétique fonctionnelle.

La subitisation requiert des objets occupant des positions distinctes et semble donc faire intervenir la capacité de notre système visuel occipitopariétal à localiser des objets dans l'espace. Elle serait un produit de l'effet SNARC (spanial-numerical association of reponses codes), étiquette forgée par Dehaene pour traduire l'observation que nombre et espace sont associés dans notre cerveau.

Une autre interprétation s'appuie sur des expériences montrant que ta subitisation implique la conjonction de deux processus présents chez les primates et à un stade préverbal chez lenfant : un mécanisme d'individuation parallèle par constitution d'index visuels (« vistral indexes ») "s7 ou de fíchiers d'objets («object files ») et de suivi des objets ("object rracking ») dans le temps ou l'espace et un mécanisme de quantification basé sur la représentation d'ensembles.

L'individuation vistelle proprement dite précède généralement l'encodage de toute autre propriété comme la localisation, la forme ou la couleur. Zenon Pylyshyn soutient que le système visuel possède un mécanisme d indexation visuelle lui permettant de détecter «les objets visuels primitifs" en tant qu 'individus indépendamment de leur localisation ou d'autres propriétés. Percevoir telle ou telle propriété suppose en effet la perception de ce à quoi se rapporte cette propriété. Ce mécanisme est primitivement lié à l'attention focale." L identité de l'objet, elle, requiert un mécanisme de suivi des objets. Selon Pylyshyn. suivre des yeux un objet est inscrit dans l'architecture du système visuel et ne nécessite pas de prise en compte visuelle des coordonnées spatio-temporelles de l'objet. La reconnaissance de l'identité d'un objet en tant qu' individu intesviendrait avant la reconnaissance de son identité en tant qu'um individu.

\footnotetext{
"(Pylyshyn. 2002). Pylyshyn fall observer tation ne peut pas subitiser des objeas non individues : des cercles concentriquess ne son pas subitisés. à l'montrise de cercles tractếs l'un à côté de l'autre,

9* (Cart'y. 2001: 2009 : Feigensum, Dehaene \& Spelkc. 2004): et auparavant (Pylyshyn \& Storm, 1988). Les concepts d'index visuel el de fichier d'nbjel sont très sinzilaires quoique nés dans des

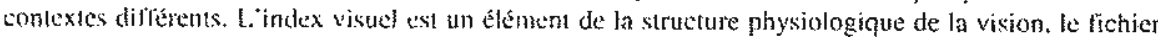

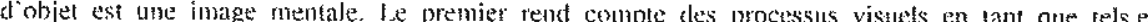
indépenchatumena des paramètres de lespace el du temps: ke second implique ces paranètres en voulignant le rôle de la thémoire de trivail.

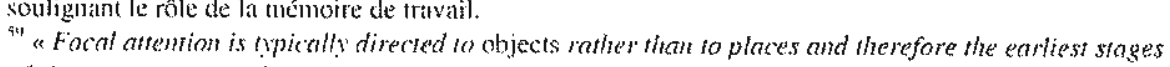

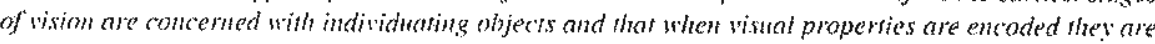
encoded as propertiess of individual ohjects $»$ (Pylyshyn. 2002. p. 4, p. 13). Sj mous royons des objets.

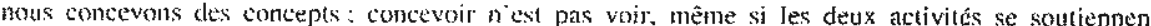
mutuelletuem.
}

Par contraste, pour de nombreux spécialistes en sciences cognitives intéressés par l'appréhension du numérique, l'individuation parallèle combine un mécanisme d'individuation visuo-spatiale et la mémoire visuelle à court terme, dont la conjonction fournirait la base de détection et de traitement des numérosités. L'individuation parallèle consiste, pour un objet présenté, en la création dans la mémoire de travail d'une image mentale d'objet, appelée

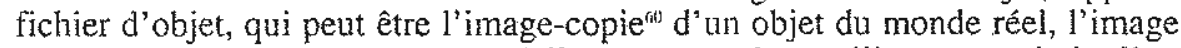
d'une pomme pour une pomme réelle par exemple, ou l'image-symbole d'un objet, par exemple $\square$ ou $\bullet$ pour une pomme ou un canard. Pour deux pommes (out deux canards) l'image mentale est par exemple $\square \square$ ou $\bullet \bullet$, pour trois pommes (ou trois canards) l'image devient $\square \square \square$ ou $\bullet \bullet \bullet$. Au-delà de trois objets, la mémoire est saturée : enfants ou adultes ne peuvent considérer simultanément plus de quatre objets."

Il est clair que l'image-symbole témoigne d'un niveau d'abstraction plus élevé que celui manifesté par l'image-copie, mais celle-ci n'est pas moins efficace que celle-là pour comprendre et agir. Reproduire et mimer n'est pas moins important que comprendre l'organisation, la structure, le sens. Scientifiques et philosophes ont du reste développé tant des conceptions mimétiques que des conceptions symboliques du monde. Du côté du mimétisme on peut mentionner la picture theory, notamment défendue par Wittgenstein dans son Tractafus Logico-Philosophicus et abandonuée dans ses Philosophical Investigations, le désir mimétique de René Girard, l'hypothèse de ligne numérique mentale de Gallistel et Dehaene, et la découverte des neurones miroirs avec l'hypothèse du «troisième cerveau», le cerveau mimétique et relationnel, de Jean-Michel Oughourian. Pour la prégnance symbolique du monde et de la pensée on peut évoquer aussi bien Husserl, Peirce, Cassirer, Freud, ou Levi-Strauss que la Gestalttheorie, les « techniques de pensée » par suites finies de signes de Hilbert, la critique radicale de la picture theory par Pylyshyn, sans parler plus largement du paradigme herméneutique présent darns nombre de disciplines.

Selon S. Carey, le mécanisme d'individuation parallèle est associé à un mécanisme de détection de l'identité mumérique, qui est physiquement fondée sur la continuité spatio-temporelle de l'objet présenté (un tas de graines n'est pas un objet individué, une voiture que l'on réduit en pièces détachées cesse d'être une voiture), même si d'autres facteurs peuvent jouer contre cette continuité (lorsqu'une personne vient à mourir, son corps devient un cadavre). Une image d'objet est créé pour une entité tridimensionnelle - éventuellement représentée par une image en deux dimensions - bornée, connexe (d'un seul tenant), qui persiste dans le temps et se meut selon des trajectoires spatiotemporelles continues.

${ }^{6}$ Il ne s'agil pas d'une photocopie ou d'un dinplicata mais d'une copje grossière ou schématique, qui ne retient pas tous les traits de la pomme réelle et qui sent d'index. Pylyshin insiste sur le fait que la vision engendre des représentations symboliques et non des duplicala des objets (Pylyshyn, 2002, p. 6-30). "Cette limite concerne aussi bien les processus sensori-motetrs que l'attention visuelle. Elle semble attester la limitation nearobiologique de la capacité à traiter des objets en parallele. 


\section{IV.I.I - Individuation parallèle et représentation analogique}

Lhypothèse d'individuation parallèle et de détection de l'identité numérique partage avec la représentation analogique non seulement Thypothèse d'une connexion très précoce sinon absolument innée entre nombre, espace et temps, mais aussi la supposition d'une représentation iconique dans notre esprit des objets ou ensembles présentés." Le format iconique des représentations mentales du noyau cognitif vaut donc aussi bien pour les ensembles discrets que pour les ensembles ou propriétés continues comme la longueur, la surface, le volume. la couleur, etc. Fornat et contenu d"une représentation sont à distinguer. ${ }^{6.7}$ Du reste, les sciences cognitives sont fondées sur le postulat initial d'un monde de représentations ou images mentales relativement autonome par rapport à la perception sensorielle et par rapport au comportement.

Cependant l'individuation parallèle, telle qu'elle est expliquée par $S$. Carey, differe de la représentation analogique linéaire de Dehaene par les caractères suivants :

1) limage mentale a un contenu numérique présent de manière seulement indirecte et implicite ${ }^{\text {th }}$, car elle met en jeu un mécanisme cognitif général de perception et de suivi des objets impliquant l'attention et la mémoire: comme l'a soutenu Pylyshyn, l'image d'un objet est d"abord une image d'objet, avant d'être l'image d'un objet. l'image de denx objets est d'abord l'mage d'objets individués occupant des positions spatio-temporelles séparées.

2) L image mentale respecte l'identité des objets, des images ou symboles différents pouvant être créés pour un ensemble d'objets différents: celá explique que dès deux mois les nourrissons distinguent entre un même objet vu dans des situations et à des moments différents et detx objets distincts.

3) Le contenu de cette image n'est pas de nature purement et simplement sensori-motrice: si elle est provoquée par un stimulus l'image n'en est pas une traduction littérale. Pylyshyn insiste beatcoup sur le fait que l'image mentale d'un objet n'est pas un duplicata mais un symbole de cet objet. De son côté, Susan Carey répète souvent que les fichiers d'objets sont constitués de "symboles" mentaux encapsulés dans un système perceptif ". mais, contrairement à Pylyshyn, elle définit les concepts comme étant des symboles mentaux constituant les unités élémentaires de la pensée, si bien qu'elle présume une activité conceptuelte symbolique de catégorisation dès le niveau perceptif, ce qui reste assez largement admis malgré les efforts principiels et méthodologiques de Pylyshyn pour caractériser de manière scientifiquement autonome le niveau de structuration perceptive

"2: (Carey. 201 1. p. 116).

to Cette distinction introduite par Zenon Pylysthyn. par exemple dans (Pylyshyn, 2003), est reprise avec insistance par $S$. Carey

ot (Feigenson, Carey \& Hatser. 2002 : Carey. 2009 ; Piazza et al.. 2011). corrélé à l'architecture anatomique de la vision.s Si tel est bien le cas et si cela indique la difficulté d'isoler en fait un niveau intuitifperceptif indépendant d'une activité conceptuelle, it est utile de distinguer, comme le fait Carey, entre concept au sens d'unité de pensée et conception, laquelle implique raisonnement, croyance, désir, explication, etc.

4) Le fait de créer mentalement un nouveau fichier d'objet pour un nouvel objet est mentalement analogue à ajouter un et préparerait ainsi la compréhension précoce d'addition ou de soustraction d'une unitét ainsi que la compréhension bien ultérieure de la fonction successeur.

5) La mémoire d'un enfant de douze mois est capable de créer au moins deux ensembles d'au plus trois objets et de comparer les ensembles par une correspondance mentale un à un ${ }^{\prime \prime}$. Les travaux d'Elizabeth Spelke ont conduit à induire la mise en ouvre dès l'âge de sept mois de cette correspondance, notamment entre stimuli visuels et auditifs qu'ils soient présentés simultanement ou de manière différéét. Or la correspondance un à un implique une sériation et un appariement ordinal d'où découle l'égalité ou l'inégalité numérique (relation pareil/pas pareil) ${ }^{\text {th }}$ des ensembles comparés mais aussi la reconnaissance de l'ensemble ayant le plus grand nombre d'éléments (relation plus grand/plus petit) ${ }^{70}$ : pour deux ensembles d'au plus trois objets chacun, par exemple deux boîtes dans lesquelles ont été introduits successivement des biscuits, des enfants de 10-12 mois choisissent l'ensemble le plus grand, c'est-à-dire la boite contenant le plus grand nombre de biscuits". L'aspect ordinal du nombre ne saurait donc être considéré comme second par rapport à son aspect cardinal et

65. Pylyshyn reconnaît l'intrication fachelle du pténomène de la vision avec divers phénomènes

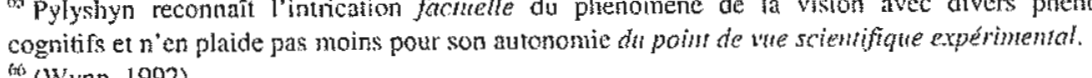

tho (Wynn. 1992).

67 (Fcigenson \& Carey, 2003).

(Spelke, 1983 ; Starkey. Spelke \& Gelınan, 1983, 1990).

(") La relation pareil/pas pareil exprime une différence, non nécessasirement une différence nunaérique. Pylyshyn a montré que l'identité et lidentité numérique sont distinctes. De plus l"identité ou la nonidentité numerique pourrait ütre reliée directennen à la perception d' ensenbles d'objets comme fotalites

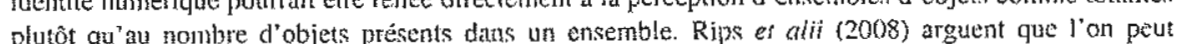

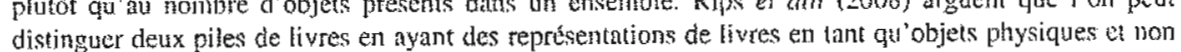
distinguer deux piles de lives en ayant des représentations de liwes en tiant qu' 'objels physiques at non des représentations de nombres (p. 633). Pourtant the variante de l'expérience de Wynn (1992) oat ton l'identité des objets et restent sensibjes à jeur identité ou non-identitê numérique.

"Selon ( Spelke, 1993) les relations statiques de forme sembient avoir peu deffet sur la perccprion de la cohtion et des contus das on 9 mois - qui percoivent potrant symétrie on f'alignenent -... tantis ces relations contmencènt à avoir un effet pour des enfants de un an et intluent fortement sur la perception adulte Par ailleurs, les circuits cérebraux pour la reconnaissance des formes et pour les numérosités sont distincts ; leur corrélation est vérifiee chez lo bébé naiss s'estompe avee l'äge. chaque circuit se développant indépendamment. Voir, par exemple. (Chinello, Cat:ani, Bontiglioli, Dehaene \& Piazza, 2013).

${ }^{71}$ (Feigenson, Carey \& Hauser. 2002) 
donc sur la base de l'extension spatiale, l'attention étant davantage portée sur" l'individualité des objets et leur volume que sur leur nombre. Cependant, la sensibilité des nourrissons à l'identité numérique de petites numérosités se manifeste préférentiellement dans le cas d'un ensemble constitué par des individus ayant des propriêtés hétérogènes. "Néanmoins certains tests semblent prendre en défaut l'idée d'une prévalence d'une représentation d'extension sur une représentation numérique pour de petits ensembles: Cordes et Brannon (2009) plaident pour une attention simultanée à l'extension et au nombre pour des petits ensembles et une préférence pour l'information numérique dans le cas d'ensembles de plus de trois éléments. Plus généralement, il est probable que le choix pour l'un des deux types de représentation réponde non seulement aux propriétés des ensembles montrés (petites ou grandes numérosités), mais aussi au protocole de l'expérimentation : entrent en jell l'âge et les attirances contextuelles des sujets soumis à l'expérience, la question pour laquelle l'expérimentateur cherche des réponses, le dispositif qu' il a mis en œuvre, etc.

La représentation par fichiers d'objets obéit donc à des principes d'individuation et d'identité numérique et implique aussi bien l'individuation selon une ou plusieurs propriétés continues que l'individuation selon l'identité numérique. Susan Carey pense que pour les petits ensembles la représentation par fichiers d'objets domine dans de nombreuses situations l'estimation approximative; elle voit donc une discontinuté cognitive entre nourrissons et adultes qui, eux, utilisent l'estimation approximative pour de petits ensernbles aussi bien que pour de grands ensembles. Cependant, comme je l'ai déjà mentionné plus haut (notes 44 et 46 ) cette conclusion a été remise en cause récemment. ${ }^{77}$ Une raison pourrait être que même dans l'estimation approximative il est nécessaire d'individuer les élements constituant un ensemble pour apprécier le nombre cardinal de leur totalité, mais cela ne peut se faire selon le même processus que pour les petits ensembles. D'autres expériences viendront certainement encore conforter soit la primauté de l'ANS et de son mode continu mimant la continuité supposée de sa cible, soit celle des fichiers d'objets et de lenrs référents individués.

\section{IV.J.2-Objet et nombre}

Si la dominance de la catégorisation d"objet sur la catégorisation numérique est encore en débat' ${ }^{7 \%}$, il est généralement admis quue la catégorisation numérique est plus fondamentale que la classification selon des propriétés

${ }^{76}$ (Feigenson, 2005 : Feigenson \& Halberda, 2004) (expériences rapportées et commentées par (Cixcy. 2009, D. 143-155): voir atssi (Haberda \& Feigenson. 2008). Cela expliquerait in difficuité des 200 . D. $143-155)$ : voir aust (Haberda nourtissons a distinguer entre tn point et deux points de meme taithe et même couleur, alors gur ils distinguent entre deux points et huit points, usant dans ce dernier cas du mécanisme d'estimation approximative $\left(X_{U}, 2003\right)$.

7 (Coubart, lzard. Speike, Marie \& Streri, 2014).

${ }^{73}$ D'après (Lourenco \& Longo, 2010) it existe primifivement un sens genéralisé de la grandeur transversal par rapport au nombre, à l'espace et au temins: "Cross-dimensional tronstor

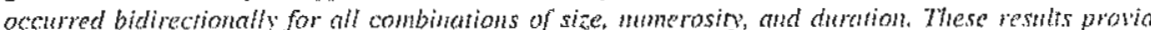

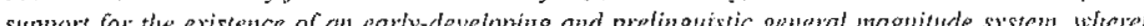
supponf for the exist representations of magnitude information are (at least partially) abstracted from the specific dimensions 》.
${ }^{71}$ Cet aspect souligné par $\mathrm{S}$. Carey est confinmé par ailleurs. par exemple par (Piazza, Fumarola. Chintllo \& Melcher. 2011 ).

${ }^{75}$ La présentation d'intrages contenant une grande quantité d'éléncnss semble induire autonatiquement une teprésentalion numérique. ce qui ne est pas le cas avec les quanités infériètures à trots. 
continues comme la forme, la taille, la couleur, la brillance, etc." Seules la continutté spatiale et ta continuité temporelle sont aussi fondamentales que l'appréhension de la numérosité comme le montre l'expérience du choix d"unt seul grand biscuit plutôt que de deux plus petits représentant en tout la moitié du plus grand. Reste à savoir si la coordination entre numérosité, durée temporelle et étendue spatiale expérimentalement attestée chez les nourrissons est le résultat d’un seul ef même mécanisme fondamental hérité de lévolution, ou si l'on a affaire à des mécanismes distincts que le nourrisson, dès ses premières heures de vie, apprend très rapidement à associer, ou encore $s$ "il $s$ agit d"une coordination acquise récemment dans l'histoire et dans une aire de culture particulière. Toutes ces options sont représentées parmi les experts.

Dans tous les cas. la numétosité est bien tenue pour une dannée primitive, qualité primaire des événements et phénomènes du monde réel ou aptitude cognitive première. Le choix finalement n'est pas entre qualité objective versus préaptitude cognitive. mais plutôt entre l'option d'une corrélation entre les denx, héritèe de l'évolution et développée par l'expérience et la créativité, option qui est celle de la majorité des spécialistes de biologie et de psychologie cognitive expérimentale. versus la position réaliste qui consiste à prêter aux nombres une existence cosmique indépendante de l'activité de l'esprit. On peut bien être à la fois intuitionniste et non réaliste comme l'est par exemple Dehaene, pour qui ales nombres sont des intuitions primitives que notre architecture cérébrale rend inévilables et que nous projetons sur le monde

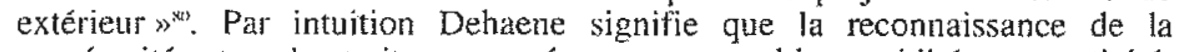
numérosité a tous les traits regroupés sous ce vocable : rapidité, altomaticité, et inaccessibilité à lintrospection consciente". Mais, outre que cette conception a ểé sévèrement critiquée par Pylyshyn (2003), il ne suppose pas que la numérosité est un phénomène objectif du monde physique indépendant de l'architecture de notre cerveau ou de nos préaptitudes cognitives. Ce qu’il entend mettre en question cest le fait de prendre le cerveau pour lin ordinateur ou une machine digitale. L'intuition est pour lui la fonction de «l'oil de l'esprit" ", et cette fonction correspond à une organjsation cérébrale non réductible au modèle computationnel qui aide à la décrite. Mais il est possible, comme je l'aj indiqué plus hant (note 56), de défendre une conception à la fois objective et historique de lintuition comme marque de l'assimilation des constructions conceptuelles.

\section{IV.2 - Quantification et langage : l'individuation parallèle enrichie}

Quine a soutenu que lat représentation d'objet en tant qu'objet n’émerge quavec la discrimination entre un, deux, quelques, plusieurs, tous, permise par lacquisition du langage entre deux et trois ans. Mathieu Le Corre et Susan Carey montrent que les nourrissons entre deux et six mois distinguent entre an et plusieurs. entre individu (singleton) et multitude d'une manière indépendante du langage. Ils invoquent un mécanisme de quantification primaire basé sur une représentation implicite densembles (set based

\footnotetext{
7" (Cordes \& Brannon. 2018, 2009, 2011 : Libertus. Starr \& B Bannon. 2013)

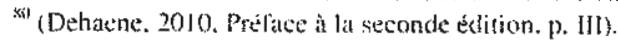

${ }^{21}$ Annuaire du Collège de France $2007-2008$. quantification system). Celui-ci serait la racine de la distinction en langage naturel entre un, deux, quelques, plusieurs, tous. ${ }^{.2}$ Combiné au mécanisme d'individuation parallèle il donne ce que Le Corre et Carey appellent l'individuation parallèle enrichie. Celle-ci créerait et stockerait dans la mémoire à long terme dés représentations pour de petits ensembles d'individus, telles que $\{\mathrm{i}\},\{j, k\},\{k, m, n\}, n^{\prime} u t i l i s a n t$ pour cela que l'individuation parallèle et la capacité préverbale de comparer deux ensembles par une correspondance un à un. Ces représentations prototypiques serviraient ensuite d'étalons pour les petites numérosités. L'individuation parallèle enrichie foumirait l'outillage conceptuel permettant de construire les principes du comptage, grâce auxquels on construit une représentation pour les entiers positifs supérieurs à trois et, par suite, une représentation du nombre en tant qu'élément de la suite gouvernée par la fonction successeur.

Tandis que pour Gallistel et Gelman (1978) et Dehaene (1997) les principes du comptage sont acquis en faisant correspondre à des symboles verbaux (un. deux, trois, etc.) ou chiffrés $(1,2,3$, etc. $)$ leur signification numélique préalablement extraite par une représentation analogique linéaire, Mathieu Le Corre et Susan Carey considèrent que l'individuation parallèle enrichie représente de manière exacte les petites numérosités de un à trois, tandis que la représentation analogique approxime les numérosités supérieures à quatre ou cinq et ne fonctionne de manière exacte en faisant correspondre un symbole à une quantité qu'après l'acquisition des principes di comptage (Le Corre \& Carey, 2007). Autrement dit non seulement représentation discrète et représentation contínue sont coprésentes dans le noyau cognitif, mais, de plus, la reconnaissance discrète par appariement un à un de deux ensembles d'au plus trois objets chacun précède largement l'acquisition du comptage et la capacité subséquente d'appréciation exacte de la cardinalité d'un ensemble quelconque - pour les petits ensembles nous avons vu que les deux paradigmes, analogique et discret, sont concurrents et que le choix de l'un d'eux dépend en partie de la manière dont est organisée l'expérimentation. En revanche l'appréhension qualitative est privilégiée pour des ensembles non constitués d'objets physiquement distincts (i.e. occupant des positions spatiotemporelles séparées), par exemple une image représentant un lapin et une voiture jouet accolés par un de leur bord, ou constitués de nombreux objets.

Ainsi l'acquisition verbale de principes de comptage n'est pas guidée par des principes préverbaux innés engendrant des grandeurs. Elle est l'cuvre d'une construction conceptuelle dont l'amorce et seulement l'amorce est foumie par des dispositions innées. Le constructivisme de Piaget est donc en partie sauvé.

On observera que Le Corre et Carey promenvent un modèle ensembliste discret différent du modèle géométrique continu de Dehaene. Ils supposent une représentation mentale d'ensemble discret, de même que Dehaene suppose une

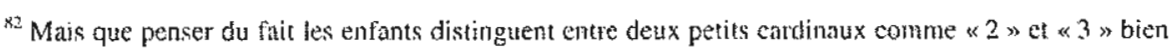
avant de distinguer "quelques-uns » de «plusieurs »? Et comment tenir compte de la remarque de Hodes (1984) que, du point de vue logique, pour spécifier un ensemble ayant exactement deux éléments la relation de non-identité $\neq$ suffit, sans gan"il soil necessaire de faire appel au nombre deux on ta la relation de non-identicé numérique? 
représentation de grandeur continue ou même de continu numérique (LNM). Dans chaque cas la configuration du modèle fournit une grille d'interprétation de phénomènes mentaux non directement accessibles et supposés universellement disponibles à la naissance. Or aussi bien la continuité numérique que la considération d"ensembles discrets quelconques sont des acquisitions récentes de la culture mathématique. Le continu numérique n'a été clairement défini qu'à la fin du XIX ${ }^{*}$ sjècle par Richard Dedekind ${ }^{\mathrm{k}}$, qui est également l"auteur, avec Georg Cantor, des concepts cardinaux de la théorie des ensembles. On peut donc se demander quelle sorte de légitimité justifie l"utilisation automatique et sans réserve préalable de cet outillage moderne à titre dhypothèse explicative de la genèse de processus mentaux dont ils sont un produit sophistiqué et dont l'usage n'est maîtrisé que par des groupes sociaux relativement restreints (les mathématiciens, les professeurs et les étudiants de mathématiques, etc.). Outre les biais produits par les types de présentation (objet isolé ou série d'objets présentés simultanément ou successivement. image statique ou dynamique, etc.) les caractéristiques du modèle guidant I'investigation et les préférences philosophiques qui orientent son choix ne déteminent elles pas l'interprétation figurée des données expérimentales? Après tout, la perception directe du monde ne livre pas davantage le continu numérique ${ }^{*-1}$ que les ensembles abstraits.

\section{V - CATÉGORISATION : OBJET, IMAGE MENTALE ET CONCEPT}

\section{V.1 - La perception du monde}

Dans une perspective contraire à l'empirisme classique, pour lequel nous $n^{2}$ avons d'abord que des sensations produites par un stimulus externe et ne construisons que très progressivenent des catégories générales, les nativistes pensent que la catégorisation émerge au cours des premiers mois de la vie et que même les nouveaux-nés possèdent des habiletés de catégorisation primitives, bien antérieures à la capacité de coordonner sensation el activité motrice (contre Plaget) et bien antérieures à l'acquisition du langage dont l'influence sera en effet clécisive ensuite (contre Quine).

Pylyshyn a mis en évidence un mécanisme primitif d'indexation visuel grâce auquel les objets du monde réel sont perçus directement comme unités indivises et identiques à elles-mèmes. Ce mécanisme est d'ordire purement visuel et déictique, il relie directement l'objet à l'neil sans la médiation du concept d'objet et sans intervention des coordonnées spatio-temporelles ni d'atutres caractéristiques physiques de l'objet. Pour Pylyshyn une chaîne causale relie lobjet réel au symbole mental correspondant qui est un fichier d'objet initialement vide) et celui-ci réfère directement à l'objet source. Le premier lien est causal; le second, de nature référentielle. est dans l'ordre de la nature de la vision. il n'a pas besoin d'être conceptuel en ce sens qu'il ne fait pas intervenir le système cognitif central à l'ouvre dans le raisonnement,

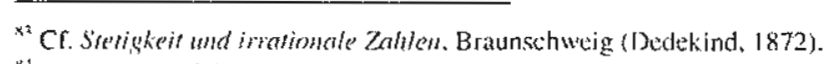

*1 Pour un mathématiciten comme (Dedekind. 1872). le continu mathómatique n'ex pas affaire d'experience visaclle mais de débmition et l'espace géconetrique est une constuction fondée sur des axinntes, non ke reflet de lexpace plysique trimensionnel. l'inférence, la décision et d'autres processus rationnels ; et il n'a pas besoin des supports ou véhicutes du nombre, de l'espace ou du temps.

Contrastant avec le structuralisme physiologique de Pylyshyn, des travaux de psychologie cognitive montrent que l'enfant possède dès avant la fin de sa première année le «concept naïf d'objet physique" qui lui fournit les premiers paramètres de l'individuation et de l'identité. La perception d'objet est antérieure à la préhension ou manipulation d'objet : elle est déjà présente chez les nourrissons entre trois et six mois. Le nourrisson perçoit prioritairement le caractère d'entité individuée connexe, qui est préservé dans le mouvement de l'objet, avant de percevoir la forme, la taille ou la couleur de l'objet. Il disposerait au niveau perceptif d'un «sortal» (c'est-à-dire d'un concept fournissant des critères d'individuation et d'identité), celui d'objet physique, défini par des critères spatio-temporels d'individuation et d'identité, avant de disposer de «sortals » plus spécifiques comme table ou personne pour lesquels les critères spatio-temporels ne suffisent pas à déterminer leur intégrité et leur identité numérique.

Quoiqu'il en soit, il faut distinguer entre perception d'objet et perception d'objet physique. Pylyshyn n'est pas le seul à soutenir que la perception d'objet en tant qu'objet n'est pas la perception d'objet physique, ni le seul à distinguer la perception proprement dite de la pré-perception anticipatrice et de la post-perception (interprétative). Le même genre d'observations est ađressé à S. Carey comme à $\mathrm{E}$. Spelke par Tyler Burge." $S$. Carey répond en précisant que, selon elle, les expériences montrent que nourrissons et adultes ont des représentations conceptulelles d'objet. que les nourrissons ont aussi une représentation d'objet physique comme entité matérielle séparée, bornée par des contours, connexe et se déplaçant selon des trajectoires continues et qu'ils ont, avant le stade du langage, un symbole mental de pluralité qu'ils appliquent aux ensembles d'objets.

Qu'elle soit considérée comme «pure perception » ou comme indissociable d'une activité pré-conceptuelle, la perception primaire d'objet en tant qu'objet montre que le monde perçu r'est pas constitué de qualités mais primitivement

4s Un objet physique est une entifé persistante, se déplaçant conme un tout conservant ses frontiêres dans le Géphlacement, ayant cohession et continuite spatio-temporelfe. (Spelke, 1985) suppose que nous disposons à la naissance d'une physique naïve sur la base de laquelic nous construisons enstite notre science physique. Cette physique naive renose sur la notion innée d'objet physique en taist gutunité persistante et identique ef elle comporte des regrésentations relatives au mowement des objels et à leurs interactions cairsales.

"Burge, 2011, in Carey, 20I1, p. 125): "If is a mistake to require of a system that has object representations that it have anantificational devices, or representations of criteria for mumerical identity, or specifications of contriutity under loss of perceptuat contact (using that "vocabulan"). Nor need object perception represent particulars as persising, ar as independent of an obsenter, or as tamperceited, in order to perceptially represen something as an object or body. A perception as of objects need not represent pessistence, observet's, perceptuat conner, or independence from obseners. Sich requirements confitse principles according to which perception operates wilh representrations that occter in perceptual object representation. I... Perceptnal representations include object (body)

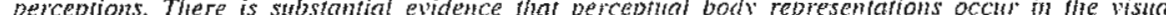
systens of many is entities as bodies are associared with very eavly vision. The anicipations are not matters of conception or prediction". 
organisé en unités séparées et persistantes à travers leurs transformations, en objets porteurs de qualités. Le flot continu de stimulations sensorielles est converti en unitês de signification (selon l'expression de Husserl) psychologiquement représentées par des symboles mentaux, précurseurs de constructions conceptuelles. La digitalisation du continu semble donc inhérente à la catégorisation perceptive. qui est le premier niveau de l'activité de catégorisation. On peut soutenir que même l'estimation approximative d'une valeur sur une ligne continue, c'est-à-dire l'estimation approximative de la valeur arithmétique d'une coupure de Dedekind, ou l'estimation globale d'une multiplicité désordonnée d"objets, un troupealu de moutons par exemple, relève d'un certain type de catégorisation. c'est-à-dire de discrétisation, et que ce type diffère à la fois du type de catégorisation individuante portant sur de petits ensembles et du type de catégorisation sérielle à l'cut vre dans la considération d'un ensemble quelconque comme collection discrète d'individus.

Entendue dans une acception large, la catégorisation, répondant à des contraintes naturelles. commence d'abord par découper visuellement des objets avant de reconnaître leur identité ou de détecter par comparaison les similarités ou dissemblances de propriêtés dobjets distincts, c'est-à-dire avant de constituer des classes d objets. ${ }^{.7} \mathrm{~L}$ 'information fournie à l'esprit par le système visuel est donc abstraite. cest-à-dire dépourvue de qualitésx", et catégorique au sens où elle consiste en « labels » ou « index » ou « fichiers d'objets » assignés à des objets du monde et ainsi préparés pour la conceptualisation. Mais elle ne nécessite pas d'avoir ou d'utiliser le concept d'objet. Pour Pylyshyn l'attention qui se focalise sur un ou plusieurs objets perçus en tant qu'objets est l'acte médiateur entre perception et cognition en même temps qu elle établit un lien direct, par indexation visuelle. entre l'esprit et le monde: un index visuel " pointe " directement vers un objet du monde. On lit sous sa plume:

"We pick ou and individuate primitive visual objects as a precursor to focusing attention on them and encoding their properties. Such vistal indexes play an important role in atuching symbols to things and they also play a rola in allowing visual images to inherit spatial properties of perceived space. $\$^{\text {*n }}$

On peut penser, comme Pylyshyn. que le clouble lien de lobjet au fichier d'objet, causal de l'objet vers le fichier d'objet et référentiel du fichier vers

\footnotetext{
${ }^{87}$ Je n'tntre pas tei duns la question de satoir si une classe ext constifuée par similarite, et si elle tst

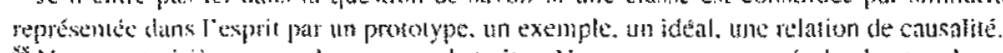

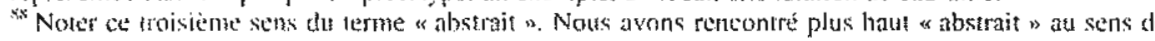

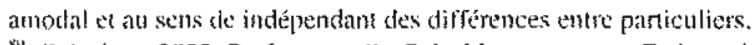

s.r (Pylyshyn. 2602. Preface, p, vii). Pylyshint est. comme Fodor, adepte d un langage mental : il est donc maturel gü il insiste à la fois sur le caractere symbolique du contens foumi par la vision fonçue conme

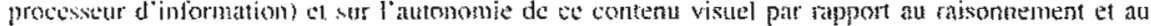

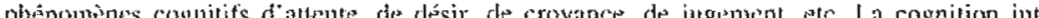

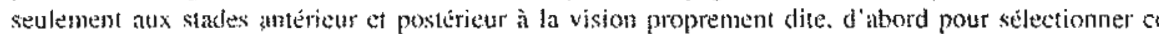

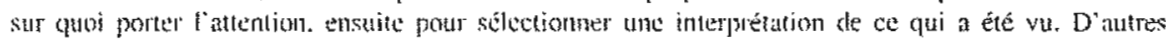

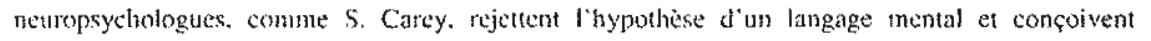
structuration de linfornation visaelle comme disseanblable de la structuration diun tangage el

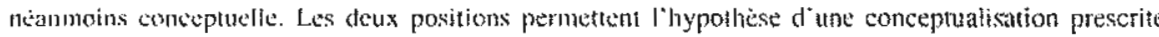
par l'arelitecture cerebale of inchendante de la connaissance.
}

l'objet, est naturel, c'est-à-dire résulte de contraintes neurobiologiques. Le l'objet, est naturel, c'est-dire to fichier d'objet débat entre naturel et conceptuel se reporte alors sur las lui-même. Index d'un objet du monde reel, un symo dichalce d'une activité conceptuelle de l'esprit au ras de la perception d'objet et d'une activite concepturelle de lesprit ad concept? Ou bien est-il seulement préalablement à toute formation partir de laquelle peut être faite une inférence et construit un concept?

Dans une theorie physicaliste et non linguistique de l'esprit comme l'est Dotamment celle de Susan Carey un concept est une représentation mentale notamment celle de s'une représentation sensorielle. Les concepts seraient un speus-ensemble de l'ensemble des représentations mentales, une représentation sous-ensemble de l'ensemble des représentations mocessus de catégorisation mentale êtant une entité existant dans l'esprit. Les processus de catégorisation et leurs corrélats, les concepts, seraient encapsulés dans les images mentalios provenant de la perception. Conceptualiser ne vient pas après la perception provenant de la perce temps qu'elle et se poursuit au-delà. Le symbole mental serait le premier acte de la pensée.

Certains théoriciens maintiennent cependant une distinction entre image et concept. Soit parce qu'ils veulent affirmer l'autonomie plysique des processus perceptifs par rapport aux processus cognitifs et associent le concept au langage (intérieur ou public), soit pour une raison de principe méthodologique, langage (intérieur ou public), soit pulyshyn, pour qui le symbole mental résulte Le premier cas est représenté par Pylyshyn, pour qui no syme copie de l'objet et d'une perception non d'une pensée ; c'est un index non une copie de lobjet et i] est une matiète à penser, non la pensée elle-même." Pylyshyn reprend le concept de «early vision » de David Marr et assume lhypothèse empiriquement argumentée d'une architecture fonctionnelle spécifique du système visuel non influencée par l'activité cognitive sur laquelle elle exerce des contraintes structurelles. ${ }^{\prime \prime}$ L' architecture du système vistel est responsable des contraintes structurelles. de la structuration de la scène visuelle. Stralleurs, d'un point de vue méthosont pas une seule et même chose. Par ailleurs, dun point dé vent on peut dologique on peut distinguer entre image et concept, plus précisément on peut à la fois reconnâtre l'intrication au niveau perceptif de mécanismes purement visuels et d'effets non linguistiques de signification tout en distinguant, ains visuels et deffets nodes ling que le fait Harold Hodes ${ }^{42}$, entre un conc

Pour ma part, il me semble qu'une unité de signification ot le symbole mental qui la représente n'est pas encore un concept ; elle ou il est seulement mental qui la represente nest pas encore un coace dans la mémoire et orvre la un contenu de representation qui laisse une trace dans la mar exemple, il faut possibilité au développement d'un concept proprement dit. Par exemple, il fau distinguer entre un nombre comme celui désigné par le mot 'quate', qui est

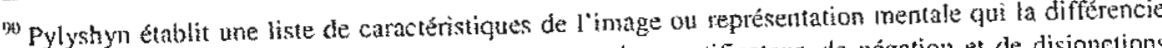
Wyla per de disjonctions

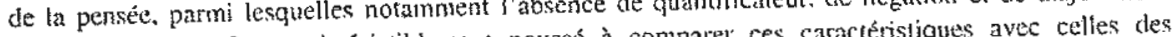

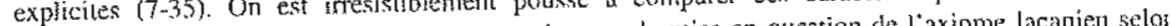

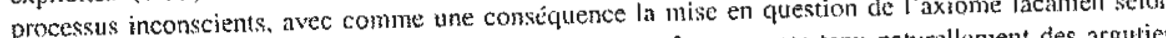

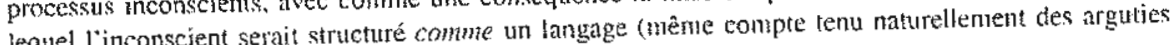
sur le « comme $)$.

sur le « comme $)$. "Larchitecture du systeme vistel peut changer sous une contuime neusus

netronale, traunatisne, etc.) mais non sous ( Asmuth. 2008, p. 657-658) 
bien un concept mumérique auquel peut correspondre l'image d'un carré ou le symbole mental $\{a, b, c, d\}$. et le concept de nombre entier auquel ne correspond l'image d'aucun nombre particulier ni même aucune image précise dans lesprit d"un enfant ou d"un adulte non familier avec les défintions structurales de l'arithmétique des nombres entiers.".3. Ainsi le concept de nombre, en tant que tel, ne dérive ni d'une ligne numérique mentale (LNM) ni d'une image mentale d'ensembles, ni de l'expérience d'objets physiques, ni de leur représentation iconique associée (object-files), ni des ressources du langage naturel. Si on voit néanmoins une issue dans l'idée que les symboles mentaux sont des concepts. on est tenu d'expliquer la différence conceptuelle entre ces concepts primitifs et les concepts de complexité supérieure, ce que tente entre autres $\mathrm{S}$. Carey par son idée de « changement conceptuel » et son hypothèse de "boorstrapping » que je n "examinerai pas ici.

Bien que la distinction entre concept nemérique et concept de nombre me paraisse essentielle, et que le passage d'une observation dobjets physiques à une conception arithmétique demeure certainement énigmatique, je n'assume atucunement l'hypothèse de Rips et al selon laquelle les enfants auraient implicitement et inconsciemment un schéma" de laxiomatique de Dedekind ou un schéma de récursion (p. 638). Outre que cette hypothèse est très peu argumentée et assortie d'ancun étai empirique, vu qu'elle assume a priori un fossé radical entre niveau arithmétique de base et niveau supérieur, j y ferai la même objection que j'ai faite au modèle analogique de Dehaene et au modèle ensembliste de Carey et que l'on peut également faire à l'hypothèse des métaphores conceptuelles de Lakoff et Núñez, ou encore à l'utilisation massive du concept de structure pour organiser les données expérimentales. Que ce soit au bétséfice d'une explication down-top (Dehaene. Carey) ou d'une explication top-dow'n (Lakoff et Núñez, Rips \& alii), c'est par une illusion rêtrospective que l'on extirpe une structure mathématique (le continu. les ensembles, les axiomes de Dedekind) du moment et du contexte historiques de son apparition pour la projeter sur une carte a-temporelle du fonctionnement de l"esprit ou sur une carte topographigue du cerveau. On notera du reste que ces modeles sont tout aussi bien mis au service de la conception d'une continuité (native ou inductive) entre perçu et conçu qua service de l'hypothèse d"une double rupture de l’arithmétịue. même sous forme rudimentaire et schématique, avec le monde plysique et avec le langage naturel. avec pour conséquence de renforcer l'innéité supposée des schémas arithmétiques.

\section{V.2 - Démarcation entre percept et concept}

Faut-il alors maintenir, de quelque point de vue, une démarcation entre percept et concept? Si oui, où la placer la démarcation? Ou bien les concepts ont-ils un pied dans la perception ef un pied dans la cognition comme le dit Neisser ${ }^{\prime \prime 5}$ ? Les constituants structuraux d'un percept sont-ils du même genre

\footnotetext{
" Voir, entre autres, (Rips. Blommineld \& Asmuth. 2008).

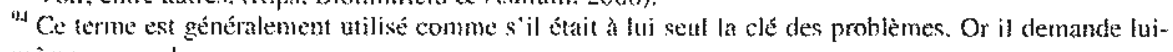
mothe the instlyse.

"Neisser. 1987 ).
}

que ceux d'un concept? N'y a-tiil pas de distinction en soi mais senlement méthodologique ou instrumentale entre percept et concept?

La difficulté de répondre mivoquement à ces questions peut être illustrée par lexemple suivant. Une psychologue (Susan Geiman ${ }^{2 \%}$ ) observe que les enfants possèdent un biais psychologique essentialiste: ils pensent que "chien" ou 'oiseau' correspondent à des catégories ou groupement de propriétés existant dans le monde réel et offrant ainsi le fondement à l'unité de signification renfermée dans le concept. D'une maniẻre générale nous croyons spontanément en lue essence des choses du monde réel visée par les processus de catégorisation. Peut-être commettons-nous ainsi une erreur métaphysique, mais c'est une erreur épistémologiquement bénéfique puisqu'elle nous conduit à catégoriser. ${ }^{27}$ Au contraire pour un neurophysiologiste (Pylyshyn) la catégorisation classificatrice est une vision généralisatrice: nous ne pouvons, dit-il percevoir une chaise présente sans percevoir cette chaise comme élément de la classe des chaises, celles-ci différant par leur forme, leur taille. la couleur de leur bois, leur garniture, etc. et occupant des lieux distincts à des moments distincts. Ce que les psychologues attribuent à la croyance, donc à un effet cognitif non critique, est rattaché au mécanisme proprement visuel par les biologistes. Pour Pylyshyn la vision est structurante et l'index causalement produit dans l'esprit par la perception d'un objet du monde est bien un symbole, mais ce n'est pas un concept proprement dit. C'est d'un point de vue similaire que C. Gauker fait observer à S. Carey qu'il ne suffit pas qu'une représentation ne puisse être expliquée en termes sensori-moteurs ni il suffit qu'elle soit intégrée à différents domaines cognitifs ou à une attitude intentionnelle pour être une représentation conceptuelle. Il y a bien sûr des représentations iconiques d'objets mais aucune représentation iconique n'est le concept d'objet." De même Tyler Burge objecte que les représentations perceptuelles d'objet ne sont pas des concepts. ${ }^{\text {*) }}$

Tenir le symbole mental pour un produtt direct de la perception et placer la médiation vers le concept dans une attitude cognitive, l'attention qui, se focalisant sur un objet du monde, déclenche lá formation du symbole mental indexant l'objet (Pylyshyn), ou considérer le symbole mental lui-même, en tant que représentation iconique mobilisant l'attention et la mémoire de travail, comme une pré-conception (Carey), dans les deux cas on reconnaît l'implication de la représentation iconique dans les processus de structuration et de conceptualisation. Si le cerveau n'est pas réductible à une machine logique et crée des ímages qui résultent d'une perception visuelle structurante et sont enrôtées dans des attitudes rationnelles de catégorisation, inférence, etc., si le flux sensoriel est d'abord discrétisé en unités indivises, les objets, qui sont simplement indexées avant d'être soumises aux filtres psychophysiques du nombre, de l'espace et du temps, si l'on prend en compte le fait que l'aire cérébrale visuelle peut être activêe non seulement par un stimulus extérieur

\%(Gelman \& Weliman, 1991).

"T) (Medin, 1989).

\%3 Concepts are not icons, in (Carey, 2011, p, 127). S. Carey répond à celte objection ent rappelant qu'il faut dissocier le contenu d'unte représentation. qui peut fort bien être par exemple le concept mêne d'objet dans la représentation en richier d'objot, de son fornat qui selon elle est toujours iconique.

(B) (Burge, 2011, in Carey, 2011. p. 125). 
mais aussi pal une représentation imaginaire non provoquée par un stimulus extérieur, alors la philosophie correspondante serait un intuitionnisme kantien naturalisé. Et c'est bien dans cette perspective qu'est proposé en 2010 par $\mathrm{S}$. Dehaene et $\mathrm{E}$. Brannon un programme de recherche. Dans son cours at Collège de France des années 2007-2008. S. Dehaene affirmait déjà :

«La position que j’ai defendue [...] postule que les fondements cognitifs des mathématiques doivent être recherchés dans une série d'intuitions fondamentales de l'espace, du temps. et du nombre, partagées par de nombreuses espèces animales, el que nous hétitons d'un lointain passé où ces intuitions jouaient un rôle essentiel à la survic. Les mathématiques se construisent par la formalisation et la mise en liakon consciente de ces différentes intuitions ».

Cette perspective intuitionniste kantienne ne coincide pas avec la perspective purement conceptuelle, créationniste et instrumentale de Richard Dedekind ${ }^{\text {ma }}$. que Dehaene invoque parfois bien qu'elle détermine un concept de nombre abstrait distinct de la représentation familière et réputée intuitive des nombres entiers, construits non dans lintuition mais dans l'entendement sans le secours de l'intuition. ${ }^{3 n}$

Il faut maintenir une distinction entre concept et image mentale sousjacente, ne serait-ce que parce fous les concepts ne sont pas associés à une image mentale. Des concepts trop abstraits ou trop hétérogêties ne donnent pas forcément lieu à une représentation imagée. ${ }^{113}$ D Weiskopf donne comme exemple le concept de justice, mais l'image d'une balance lui étant culturellement associée. le concept de vertu est un meilleur exemple de concep sans image car ne découlant pas d"une perception d'objet. Il n y a d'ima non plus pour le concept de mobilier qui renvoie à des objets du monde mais groupe des éléments anssi dissemblables qu'une chaise et un miroir. L'idée qu'il in existe pas de format unique pour toutes les représentations mentales qui sont des concepts mérite d'être examinée. Pour Weiskopf différents modes de représentation sont mis en cuvre en fonction du type d'information encodée, de la manière dont elle est acquise. et des attitudes inférentielles, c'est-à-dire de ce que l'on se propose de faire. La variété de représentations vaut aussi bien pour les percepts que pour les concepts. $\hat{A}$ un concept correspond non pas un seul type de représentation mentale (mimétique, iconique, linguistique, etc.), mass un ensemble structuré de représentations mentales de divers types.

De ce point de vue, possëder un concept renvoie non pas à une image mentale mais à une aptitude (partiellement innée et fortement dévelopoée par l'exercice). l"aptitude de se représenter une catégorie indépendamment de son mode de présentation (iconique ou non) et des attributs qu'elle est supposée fecouvrir. Telle est par exemple l'aptitude de se représenter un objet en tant

${ }^{\text {itan }}$ Le programme de (Dehateme \& Brannon. 2010 ) cherche à acomprendre comment naissent les intutions bondantentales et conment eftes sont reliées à leurs mécanismes neuraux associés et à déterminer lesquelles de ces intuitons sont independnotes de l'expérience er lesquedless sont enrichies par l'exercice et l'Édticrtion "

i"it (Dedekind. 1888).

"W" (Delaresze. 2010. Preface ò la seconde exdition. p. [II)

ims (Weiskopf. 2008) qu'objet, indépendamment de sa forme, de sa situation spatio-temporelle, de sa couleur, de ses rapports à d'autres objets semblables ou dissemblables.

Parler d'aptitude, c'est dire que le concept n'est pas une entitê mentale, mais le produit d'une fonction mentale reliée à une information sur la manière dont les objets sont perçus, se comportent, sont utilisés. Envisagés sous cet angle, les concepts sont fortement contextualisés; ils sont integrés dans des schémas ou structures conceptuelles fondées sur des principes fonctionnels d'organisation. Cette idée n'est pas notvelle en psychologie et a été remise à l'ordre du jour sous le vocable de schéma dans les années 70 . Désormais, le concept de schéma est évoqué pour caractériser la structure des représentations d'événements statiques, d'objets (Le Ny, 1989, 2005 ; Rumelhart \& Norman, 1981), de situations (Bryant, Tversky \& Franklin, 1992; Mandler, 1979 , 1983), d'événements dynamiques, et d'actions (Nelson, 1977; Schark \& Abelson. 1977 ; cf Jagot, 2002, pour une recension critique). Dans les schémas, les entités sont liées entre elles parce qu'ensemble elles concourent à remplir une certaine fonction. Les schémas sont stockés dans la mémoire à long tertne et servent de modèle ou de cadre pour analyser, structurer, interpréter et utiliser activement des informations nouvelles. Nous en avons rencontré un exemple dans le symbole mental d'ensemble associé à

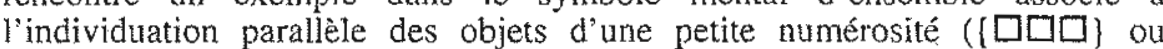
$\{i, j, k\})$, un autre dans l'hypothèse de la métaphore conceptuelle. Inutile de souligner ce que le concept de schéma doit à la mathématique modene.

La théorie fonctionnelle et dynamique du concept ${ }^{\text {th }}$ a le mérite de souligner l'existence d'une connaissance conceptuelle des objets fondée non seulement sur une analyse interne des propriétés de l'objet mais aussi sur l'analyse des relations externes de cet objet. Les structures formées sont plus flexibles, car les propriétés à la base de la sinulitude impliquée dans la formation d'une catégorie sont diversifiées et sélectionnées en fonction du contexte ou de l'intention de l'agent.

Mais elle laisse par contre inchangé le postulat que les processus de formation des concepts - fondamentalement inductifs-portent sur des représentations symboliques, complexes, sémantiquement interprétables. Ne peut-on en rendre compte par de simples mécanismes associatifs agissant sur des données élémentaires (issues de la perception) fortement connectées, et par une mise en commun de multiples traitements locaux ? Une catégorisation sans représentation?

\section{CONCLUSIONS}

J'ai commencé cette étude par la patiente lecture d'une large moisson de publications de neurobiologistes et de psycliologues du développement, dans l'espoir de recomposer, à partir des résultats éparpillés, parcellaires et sans cesse à nouveau subdivisés et stratifiés, des lignes de direction pour une philosophie contemporaine de la science. J'ai acquis ainsi queloue familiarité avec une petite fraction du domaine fascinant et incroyablement foisonnant des neurosciences, celle qui concerne les nombres. J'en ai saisi quelques

Int J'ai montré ailleurs (Benis Sinaceur, 2014) tout ce çue l"inalyse de la catégorisation concepuelle doit aux processus. inmmémoriaux ou plus récents. de l'abstraction mathématique. 
bifurcations historiques et quelques oscillations de l'empirisme au rationalisme, dans leurs versions modernes naturalisées. La plupart des scientifiques soulignent le caractère expérimental de leurs recherches, dénué veulent-ils d'a priori idéologique. de parti-pris philosophique et de preuve déductive logique. Rien que des hypothèses, des observations, des expérimentations en si multiples variantes, des inductions, des propositions ou thèses spéculatives qui sont des extrapolations et des rationalisations de résultats expérimentaux censés tradurire des faits. Tout un matériau distribué en modèles destinés à expliquer des faits, à réformer nos vues philosophiques sur la nature et l'origine des nombres, et à adapter nos principes éducatifs pour faciliter l'acquisition de performances arithmétiques. Ces modèles pourtant sont tributaires de points de vues correspondants à une préférence philosophique ou à un état du développement de la science et de la culture caractérisé par l'abondante utilisation des structures comme outil d"investigation, d"organisation et d"analyse des données (structures gestaltistes des formes, structures physico-géonétriques du mouvement, structures ensemblistes et fonctions de transfert, et d'autres encore). Les derniers acquis de la science sont retrouvés dans les aptitudes tenues pour innées à l'état inchoatif. C'est évidemment la façon la plus « rationnelle» et en tout cas la plus directe de raccorder nos constructions conceptuelles à nos données naturelles en rapportant à l'évolution la cause de leur adéquation. "I"'

Cette circularité entre le construit et le donné, entre les faits et leurs explications n'étonnera pas un philosophe, elle est l'indice de la tenace illusion de l'origine et de l'espoir insensé mais si fécond qui la conforte, l'espoir de surprendre le commencement ou le «fondement ultime " de nos pensées et de nos actes. Si la neuropsychologie et les sciences cognitives développent de nouveaux champs d'investigation, fourmillant d'aperçus inédits et de pistes de réflexion en elles-mêmes stimulantes, est-il besoin de leur prêter des lettres de noblesse en leur octroyant le privilège de fonder la connaissance? Non, car la force d'une théorie ou d'une discipline est d'avoir ou de placer sa raison d'être dans son dynamisme propre.

Du tournant cognitif. je retiens labandon du primat du langage et une certaine distance par rapport aux postulats logico-linguistiques de Chomsky et de Fodor et. plus généralement, à ceux de la philosophie analytique. Cependant. les analyses proposées des états mentaux suivent des modèles computationnels ou des schémas syntaxiques $\mathrm{y}$ compris pour expliquer la manipulation des images engendrées par la perception ou les mutations sémantiques (le boorstrapping tel qu'il est expliqué par S. Carey combine l'idée démergence à des concepts logico-structuralistes comme celui de "placeholder »). D'un côté, les cognisciences nous confirment qu'it y a de la pensée avant l'acquisition du langage, de la pensée visuelle (visual thinking) notamment. mais d'un autre côté, la pensée avant le langage, si elle a une expression visuelle, sa structure n'en demeure pas moins celle d'un langage puisqu'elle combine des signes, index ou symboles. Pensée et langage

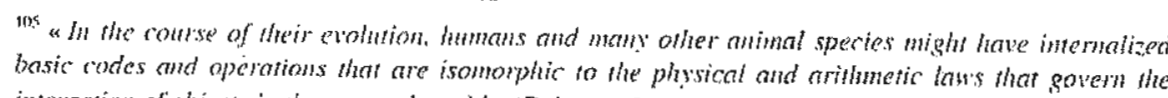
interaction of objects in the extemal trorld o (Dehaene \& Brannon, 2010. p. 517 ). fonctionnent quasi-pareillement ou, du moins, sont soumis aux mêmes outils d'analyse.

Plusieurs auteurs - mais pas tous - insistent sur le fait que nos images mentales ne sont ni des photocopies ni des tableaux des objets et des événements du monde réel. Pour eux, contrairement à ceux qui plaident pour une perception analogique du monde fondée sur l'adéquation installée par l'évolution (Dehaene notamment), les images mentales sont des symboles (Pylyshyn, Carey); leur contenu est plus ou moins arbitraire mais il a une référence dans le monde et une signification pour l'esprit.

Les représentations ou images mentales sont-elles la naturalisation des schèmes au sens kantien, comme pourrait le suggérer le programme de S. Dehaene et $\mathrm{E}$. Brannon?

Il me semble d'abord que ce pourrait être le cas dans la conception symbolique plutôt que dans la conception analogique des reptésentations mentales. Car le schème kantien n'est pas une image mais un procêdé général pour représenter un concept dans une image. Voici un passage qui illustrera ce propos:

(Le schèrne est toujours, en lui-mềme, un simple produit de l'imagination; mais étant donné que la synthèse [souligné par moi] qu'accomplit cette dernière n'a pour objectif aucune intuition particulere, mais seulement a determination de la sensibilité, le schème doit cependant toujours être distingué de l'imatge. $\rangle^{i n g}$

Ensuite, même dans le cas d'une conception symbolique des représentations mentales il resterait a préciser les différences avec le système kantien. L'une d'elles est patente : les représentations mentales surgissent de la perception et sont materiellement catsées par elle, tandis que les schèmes kantiens sont «les vraies et uniques conditions [souligné par moi] qui permettent de procurer [aux catégories ou concepts purs de l'entendement] une relation à des objets, par suite une signification. [Bedeutung, souligné par Kant] "'m?. Les schèmes kantiens ne sont pas le produit de l'intuition mais les "règles générales de la synthèse " "est-à-dire de la détermination de notre intuition conformément à un concept pur de l'entendement restreint dans son usage par les conditions formelles de la sensibilité (l'espace et le temps) Chaque catégorie a un schème: le schème des grandeurs ponr le sens externe est l'espace, le schème de tous les objets des sens en général est le temps, le schème de la quantité est le nombre, le schème de la substance est la permanence du réel dans le temps, etc. Sans schème il n'y pas de représentation d'objet mais seulement des concepts de l'entendement, c'est-àdire selon Kant de simples unités logiques des reprësentations. Le schène kantien n'est pas la représentation. Ni son format ni son contenu. Mais la règle

${ }^{106}$ (Kant, 1781, Deuxième partie: La logique transcendantale, Analytique transcendantale, $2^{2}$ livre, chapitre 1, trad. A. Renaut. Panis. Aubier, 1997, p. 225-226).

${ }^{167}$ (Kant, 1781, ibid, trad. A. Renaut. Parrs, Aubier, 1997, p. 229). Selon notre usage actuel hérité de la division établie ensuite par Frege entre 'Simn' et 'Bedeıtrmg' il faudrait traduire par téférence. Mais on peut aussì comprendre « signification » au sens modète-thêorique de « réalisation ».

${ }^{108}$ Souligné par moi 
générale qui rend la représentation possible. La question qui se pose alors est de savoir dans quelle mesure la substitution d'un symbole (la représentation mentale effective et variable) à une règle fixe et générale (le schème kantien) préserve l'architecture cognitive kantienne par un simple transfert des structures formelles kantiennes sur les structures neurales on neuropsychologiques.

\section{RÉFÉRENCES}

Anderson, M.L. (2010). Neural Reuse: A Fundamental Organizational Principle of the Brain. (Target anticle). Behavional and Brain Sciences, 33(4).

Ansari, D. \& Dhital, B. (2006). Age-related Changes in the Activation of the Intraparietal Sulcus during Nonsymbolic Magnitude Ptocessing: An Event-related Functional Magnetic Resonance Imaging study. Joumal of Cognitive Neuroscience, $18(1), 1820-1828$

Benis Sinacelr, $H$.

(1993). Du formalistne à la constructivité: le finitisme. Revue imternationale de philosophie, yol $47.14^{\circ} 186,4 / 1,251-283$.

- (1994). Calculation, Order and Continuity. In Ph. Ehrlich (éd.) Real numbers, Generalizations of the Reals, and Theories of Continut (pp. 191-206). Dordrecht, the Netherlands, Kluwer Academic Publishers.

(1994). Jean Cavailles. Philosophie mathématique. Parts, Presses Universitaires de France.

- (1995). Formes el concepts. In J. Proust \& E. Schwartz (éds.), La connatssance philosophique. Essais sur laame de Gilles-Gaston Granger. Paris, Presses Universitaires de France, 93-120.

- 2000). L'interpretation en mathématiques : de Descartes à Hilbert et Tarski. In P. Radelet-de Grave \& J.F. Stoffel (éds). Les uenfants maturels» de Descartes. Brepols. 205-221.

(2006). From Kant to Hilhert: French Philosophy of Concepts in the Beginning of the XXth century. In J. Ferreitós \& J.Gray, The Archifecture of Modem Mathematics, Oxford University Press, 349-376.

(2010) Nominalisme ancien. sontes entités mathénatiques sontelles des criations de notre esprit? In Constriction. Festschriff for Gerhard Heimanom. Tributes Series Editor Dov Gabhay. 26!-276.

- Cavaillès. Paris. Les Belles Lettres.

(2014). Facets and Levels of Malhematical Abstraction, Philosophia Scientie, 18 (1). $81-112$.

- (2015). Is Dedekind a Logicist? Why such a Question Arises? In M. Panza. (éd.) Finctions and Generalits of Logic. Reftections on Dedekind's and Frege's logicisms. Springer. (Sous presse)

Burge, T. (2011). Border Coossings: Perceptual and Post-perceptual object Representation. In S. Carey, Précis of The Origin of Concepts, Behaviaral and brain sciences, 34. 125.

Butterwortls. B. (1999). The Mathenatical Brain. Londres, Macmillan.

Carey, $\mathrm{S}$.

- (2009). The origin of concepss. Oxford Universily Press

- (2011). Précis of The Origin of Concepts. Behavioral and Brain Sciences, 34

Carey S. \& Xu. F. (2001). Beyond Object-liles and Object Tracking. Infants Representations of Objects. Cognition. 80.179-213.

Chinello, A., Catlant, V., Bonfiglioli, C., Dehaene, S. \& Piazza, M. (2013), Objects Numbers, Fingers, Space: Clustering of Ventral and Dorsal Functions in Youn Children and Adults. Developmental Science. 16 (3), 377-393. Miny 2013.
Cordes, $\mathrm{S}$ \& Brannon, $\mathrm{E}$

(2008). The Difficulties of Representing Continuous Extent in Infancy: Using Number Is Just Easier. Child Development, Vol. 79, $N^{\circ} 2,476-489$, March/April 2008.

- 2009). Crossing the Divide: Infants Discriminate Small From Large Numerosities, Developmental Psychology, American Psychological Association 2009, Vol. 45, No. 6, 1583-1594

(2011). Attending to One of Many: When mfants Are Surprinsingly Poor at Discrimuating an Item's Size. Frontiess in Psychology, April 2011, vol, 2, article 65, www.frontiersin.org.

Coubart, A., Lard, V. Spelke, E. Marie, E.S. \& Streri, A. (2014). Dissociation Between Small and Large Numerosities in Newborn Infants. Developmenal Science. 17, p. 11-22.

Dedekind, R. [1872]. Stetigkeil and irrationale Zahlen. Brannschweig. Édition française par H. Benis Sinaceur dans Richard Dedekind, La créction des nonbres. Paris, Vrin, 2008.

Dedekind, R. [1888]. Was sind athd was sollen die Zahlen? Braunschweig. Édition francaise par H. Benis Sinaceur dans Richard Dedekind, La création des nombres. Paris, Vrin, 2008

Dehaene, $\mathrm{S}$

- (2001). Précis of The number Sense. Mind \& Language, vol. 16, 1, 16-36.

- (2008). Cours du Collège de France,

http:/citeseerx.ist.psu.ech/viewdoc/download?doi=10.1.1.4.1207\&rep=rep1\&typ e=pdf el le résumé substantiel publié cans l'Annuaire du Collège de France, $\mathrm{n}^{\circ} 108,2007-2008$.

- (2010). "La bosse des mahis. * 15 ans après. $2^{2}$ éd. revte et augmentée (premiêre edition 1997). Paris, Odile Jacob.

- 2011 The Namber Sense. How the Mind Creates Mathematics, $2^{\text {sed }}$ erevue el augmentée. Oxford Universily Press.

Dehaene, S. \& Brannon, E. (2010). Special Issue on Space, Time, and Number. Trends in Cognitive Sciences, Vol. 14, No 12,517-519.

Dehaene, S. \& Cohen, L

- (1991). Two Mental Calculation Systems: A Case Sudy of Severe Acalculia with Preserved Approximation. Neuropsychologia, 29, 1045-1074.

- (1997). Cercbral Pathways for Calculation: Double Dissociation Between Rote Verbal and Quantitative Knowledge of Arithmetic. Cortex, 33, 219-250.

Diester, I. \& Nieder, A. (2007). Semantic Associations Between Signs and Numerical Categories in the Prefrontal Cortex. PLoS Biology, $5(1 /)$ e294, 2684-2695.

Feigenson, L. (2005). A Double Dissociation in Infants' Representation of Object Arrays. Cognition, $95, \mathrm{~B} 37-\mathrm{B} 48$.

Feigenson, L. \& Carey, S. (2003). Tracking Individuals via Objectwiles: Evidence fron Infants' Manual Search. Developmental Science, 6:5, 568-584.

Feigenson, L., Carey, S., \& Hauser, M. (2002). The Representations Underlying Infants' Choice of More: Object-files Versus Analog Magnitudes. Psychological Science, 13, 150-156.

Feigenson, L., Carey, S. \& Spelke, E. (2002). Infants Discrimination of Number vs, Continuous Extent. Cognifive Psychalogy, 44, 33-66.

Feigenson, L., Dehaene, S. \& Spelke, E. (2004). Core Systems of Number, Trends in Cognitive Science, 8 (7), 307-314.

Feigenson, L. \& Halberda, J. (2004). Infants Chunk Object Anays into Sets of Individuals, Cognition, 91, 173-190.

Feigenson, L. \& Spelke, E. (1998). Numerical Knowledge in Infancy: the Number/mass Distinction. Poster Presented at the International Society for Infant Studies, At]anta, Georgia. 
Gallistel, Ch, \& Gelman, R

(1978). The Child's Understanding of Number.Cambridge, Mass. Harvard University Press.

- (2000). Non-verbal Numerical Cognition: From Reals to Integers. Trends in cogrimive Scicnce. 4(2), 59-65.

- (2005). Mathematical Cognition. In K. Holyoak \& R. Morrison (éds). The Cambrige Handbook of Thinking and Reasoning (pp. 559-588). Cambridge University Press

Gauker, C. (2011). Concepts are not Icons. In S. Carey, Précis of The Origin of Concepts. Behavioral and Brain Sciences, 34, p. 127.

Gelman, S. \& Wellman. H. (1991). Insides and Essences: Early Understandings of the Non-obvious. Cognition. 38.213-244.

Goodwin. B.C. (1993). La genèse de formes dynamiques: l'organisme et l'esprit. Inellectica. $16,45-60$

Gopnik, A. (2003). The Theory Theory as an Alternatjve to the Innateness Hypothesis In L. Antony \& N. Homstein (éds.). Chomsky and his Critics. New York. Basil Blackwell.

Gordon, P. (2004). Numerical Cognition without Words: Evidence from Amazonia. Science, 306, 496-499.

Halberda. ). \& Feigenson, L. (2008). Set Representations Required for Acquisition of the Nalural Number Concept. Behaviord and Brain Sciences, 3I(6), 655-656.

Hamad. S. (1987). Psychophysical and Cognitive Aspects of Categorical Perception: A Critical overview. Hantad. S. (ed.) Categorical Perception: The Groundwork of Cognition. Clapter I. New York, Cambidge Universily Press.

Harvey. B.M., Klein, B.P. Petridou, N. \& Dumoulin, S.O. (2013). Topographic Representation of Numerosily in the Human Parietal Coftex. Science, 341, 6, 1123 1126. September 2013.

Hodes, H. (1984). Logicism and the Onological Commitments of Arithmetic. Jormal of Philosophy. 81. 123-149

Hodes. H. (2008). On Some Concepts Associated with Finite Cardinal Numbers, in Rips et al., From numerical concepts to concepts of number, Behavioral and brain sciences $31,657-658$

Hubbaid, E.M. Piazra, M.. Pinel, Ph. \& Dehaenc, S. (2005). Interactions Bewween Number and Space in Parietal Contex. Nature Reviens Neuroscience. 6, 435-448. (Junc 2005).

Hydc. D.. Boas. D. Bhir. C. \& Carey, S. (2010). Near-intrared Spectroscopy Shows Right Parietal Specialization for Numbers in Pre-verbal Infants. Neuroinage. 53(2) $647-652$.

Hyde. D. \& Spelke, E.

(2009). All Numbers are not Equal: an Electrophysiological Investigation of Small and Large Number Representations. Joumal of Cognifive Neuroscience. S\%.(6), 1039-1053.

(20I1). Netral Signatures of Number Processing in Human Infants: Evidence for Two Core Systems Underlying Numerical Cognition. Developmental Science. $14(2), 360-371$.

Izard, V.. Dehaene-Lambertz, G. \& Dehaene, S. (2008). Distinct Cerebral Pathways for Object Identity and Number in Human Infants. PLoS Biology, 6 (2), 275-285.

Frard, V.. Pica. P.. Spelke, E., Dehaene, S. (2008). Exact Equality and Successor Function: Two Key Concepts on the Path Towards Understanding Exact Numbers. Philosophical Psycholog1: Vol. 21, No. 4, 491-505, August 2008.

lzard, V.. Sann, C. Spelke. E.. \& Streri. A. (2009). Newborn Infants Percelve Abstract Numbers. Procectings of the National Academ of Sciences, 106(25), 10382 10.385
Kant I (1997). Kritik der reinen Vernunft. Hamburg, Felix Meiner, 1956; trad. A. Renaut, Paris, Aubier [1781].

Lakoff, G. \& Núñez, R. (2000). Where Mathenatics Comes from: How the Embodied Mind Brings Mathematics into Being. New York, Basic books

Lecorre, M. \& Carey, $S$

(2005). One, Two, Three, Four, Nothing More: An Investigation of the Conce

- (2008). Why the Verbal Counting Principles are Constructed out of Representations of Small Sels of Individuals: A reply to Gallistel. Cognition, 107. $650-662$

Lécuyer R (2002). Inné fable?. Intellectica, 34, 2002/1. Introduction au volume "Débats actuels sur la cognition chéz le bébé \#

Wwatlectica oro/SiteArchives/archives/n34/34_I Introduction.pdf.

Wwo (2012). Aux sources de la connaissance. L'état du débal constructivisme/nativisme chez le très jeune enfant. Devenir, 24(3), 181-214.

constructisne/nation, A. Brannon, E. (2013). Number Trumps Area for 7-Month-Old Infants. Derelopmental Psychology, Advance online publication. dos: $0.1037 / 90032986$

Lourenco S. \& Longo M (2010) General Magnitude Representation in Human infants. Psychological Science, $2 /(6), 873-881$

Mechner F. (1958) Probability Relations within Response Sequences under Ratio Reinforcement. Jounal of the experimental analysis of behaviout, $l, 109-121$

Medin, D.L. (1989). Concepts and Conceptual Structure. American Psychologist, 44, 469-1481, December 1989

Ueisser (1987). Concepts and Conceptual Development: Ecological and Intellectual Factors in Categorization. Cambridge University Press.

Nieder, A. (2005). Counting on Neurons: the Neurobiology of Numerical Competence. Nature Reviews-Neuroscience, 6(3), 177-190.

Nieder, A. \& Miller, E.

- (2003). Coding of Cognitive Magnitude. Compressed Scaling of Numerical Information in the Primate Prefrontal Cortex. Neuron, $37(\mathrm{l})$, 149-157.

(2004) A Parieto-fronal Network for Visual Numerical Information in the Monkey. Proceedings of the National Acadeny of Sciences, USA. 101 (19), 74577462 .

Núnez, R. (2011). No Innate Number Line in the Human Brain. Joumal of CrossCultural Psychology, 42, 651-668

Piazza, M. Fumarola, A. Chinello, A. \& Melcher, D. (2011). Subitizing Reflect Visuo-spatial Object Individuation Capacity. Cognition, $[21(1), 147-153$.

Pica P. Lemer, C Iard V \& Dehaene,S. (2004). Exact and Approximate Arithmetic in an Amazonian Indigene Group. Science, 306, 499-503.

Pica $\mathrm{P}$ Mckrink $\mathrm{K}$. Snelke, E. \& Dehaene, S (2013) Non-symbolic Halving in an Amazonian Indigene Group. Developmental Science, 1613), 451-462.

Platt I. \& Johnson, D. (1971). Localization of Position within a Homogeneous Behavior Chain: Effects of Error Contingencies. Leaming and Motivation. 2, 386 414.

Pylystyyn, Z.W. (1999). Is Vision Continuous with Cognition? The Case for Cognitive Impencubatily of Visual Perception. Behavional and Brain Sciences, 22, 341423.

- (2002). Seeing and Visualizing.

hitp/lruccs.rutgers.edu/faculty/pylyshyn/bookall.pdf.2002

- (2003). Return of the Mental Image: Are there Really Pictures in the Brain? Trends in Cognitive Sciences, Vol.7, Na3,113-118, March 2003.

htep:/ruccs.rutgers.edu/laculty/pylyshyn/tics_imagery.pd/ 
Pylyshyn. Z. W. Storm. R. W. (1988). Tracking Multiple Independent Targets: Evidence for a Paraltel Tracking Mechanism. Spatial Vision, 3, 179-197. hup://ruces.rulgers,cdu/hp/puth/papers/stoming pdi

Revkin. S.. Piazza, M.. Izard, V., Cohen, L. \& Dehaene, S. (2008). Does Subitizing Reflect Numerical Estimation? Psychological Science, 19(6). 607-614.

Rips, L., Blommfield. A., \& Asmuth. J. (2008). From Numerical Concepts to Concepts of Number. Behavional and Brain Sciences, 31, 623-687.

Roitman. J., Bramion, E., \& Plall. M.

(2007). Monotonic Coding of Numerosity in Macaque Lateral Intraparietal Area. PLOS Biology, $5(8)$ c208, $1672-1682$.

- (2012). Representation of Numerosity in Posterior Parietal Cortex. Fronters in Integrative Nenroscience, vol. 6. May 2012.

Simon, O. Mangin, J.F., Cohen, L., Le Bihan, D., \& Dehaene, S. (2002), Topographical Layout of Hand, Eye. Calculation, and language-related Areas in the Human Parietal Lobe. Netron, 33(3). 475-487.

Spelke, E.

- (1983). Cognition in Infancy. MIT Occasional Papers in Cognitive Science. $N^{\circ} 23$.

- (1985). Perception of Unity, Persistence, and Identity: Thoughis on Infants Conceptions of Ohjects. In J. Mehler \& R. Fox (éds.), Neonale Cognition. Hilssdale. NJ. Erlbaum.

- (1993). Gestalt Relations and Object Perception: a Developmental Study. Perceprion, 22, 1483-1501.

- (1998). Nativism, Empiricism, and the Otigins of Krowledge. Infant Behavior and Developnient. 21, 18I-200.

Spelke, E.. Lzard, V.. Coubart, A., de Hevia, M.D. \& Strer, A. (2014). Representations of Space. Time, and Number in Neonates, Proceedings of the National Academy of Scicnces. $/ / 1 /(13), 4808-4813$

Starkcy, P., Spelke. E. \& Gelman, R.

- (1983). Delection of Intermodal Numerical Correspondences by fuman Inlants. Science. 22, 179-181.

- (1990). Numerical Abstraction by Human Infants. Cognition, 36, 97-127.

Starr A., Libertus M. \& Brannon E. (2013). Infants Show Ratio-dependent Number Discrimination Regardiless of Set Size. Infancy. $1-15$.

Stewarl, I. (1993). Au-delà de linné et de Yacquis. Intellectica, 16, 151-174.

Weiskopf. D.

- (2008). First Thoughts. Philosophical Psichology, 2/(2), 251-268

- (2009). The Plaradity of Concepts. Symhese. 169, 145-173.

Wynn, K.

- (1992). Addition and Subtraction by Human Infants. Noture, 358. 749-750.

- (1996). Infants' Individuation and Entmeration of Actions. Psychological Science. 7. 164-169.

(1998). Psyctological Foundation of Number: Numerical Competence in Human Infants. Treneds in Cogmitive Sciences, 2, 296-303.

$X_{u}, F .(2603)$. Numerosity Discrimination in Infants: Evidence for Two Systems of Representations. Cognition. 89. B15-B25.

Xu. F. \& Carey S (1996). Infants' Metaphysics: the Case of Numerical Identity. Cognitive Psychologi: 30,111-153

Xu. F. \& Spelke. E. (2000). Large Number Discrimination in 6-month-old infants. Cognifiom.74(1), B I-B11.

\section{intellectica}

revue pluridisciplinaire de sciences cognitives

Appel à confributions

intellectica est la revue de l'Association pour la Recherche Cognitive. Elle sadresse à l'ensemble des disciplines intéressées par l'éiude de la cognition.

intellectica publie des travaux théoriques ou expérimentaux originaux, ainsi que des travaux de synthèse, des rapports de recherche et des notes critiques. Elle que des théries, modeles et architectures associés aux structures et aux raite des theories, modeles et are hatre appréhendés sous leur forme stable ou processus cognitifs. Ceux-ci peuvent éte apprehendes ou en liaison avec leurs dans leur développement, décrits abstraitement

déterminations biologiques, physiques ot sociales.
La revue aborde notamment la perception, la motricité, le langage, le Laisonnemen formes de l'intentionualite, dans leuts modalités individueles e raisonnenes, naturelles ou artificielles. Elle s'intéresse aux logiques, aux collectives, naturelles ou artificiel modèles neuro-mimétiques et dynamiques, représentations des connaissances, aux modeles neuro-nications dans des domaines aux systèmes adaptatifs. Elle présente aussi des applications dans des domatique. tels que l'education, l'ergonomie, l'interaction homme-machine et la robotique tels que anime enfin les débats épistémologiques, sur la base d'un pluralisme radical dans le choix des auteurs et des disciplines, comme dans celui des themes, des écoles, ou des paradigmes.

Les propositions d'articles ou de dossiers thématiques sont à adresser an rédacteur en chef, Olivier Gapenne : soumission@intellectica.org.

Chroue proposition d'article (assortie de résumés en anglais et en français) est examinée par au moins deux rapporteurs internes et deux rapporteurs externes, de domaines disciplinaires différents. La revue publie exclusivement des travaux à

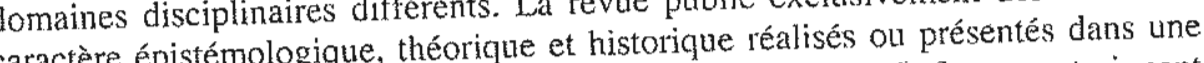
caractère épistémologique, theorque et histon le contenu ou la forme restreignent perspective interdisciplinaire. Les articles dont le contencianche crédités d'une priorité plus faible.

La revue intellectica est publiée avec le concon's da Centre National du Live, de l'Universite Compiegne ef de l'Institul des Sciences de la Communicarion du CNRS 